\title{
IUCrJ
}

Volume 5 (2018)

Supporting information for article:

Double role of metalloporphyrins in catalytic bioinspired supramolecular metal-organic frameworks (SMOFs)

Arkaitz Fidalgo-Marijuan, Eder Amayuelas, Gotzone Barandika, Edurne S. Larrea, Begoña Bazán, Miren Karmele Urtiaga, Marta Iglesias and Maribel Arriortua 


\section{Double role of metalloporphyrins in catalytic bioinspired supramolecular Metal-Organic Frameworks (SMOFs)}

Arkaitz Fidalgo-Marijuan ${ }^{\text {ab }}$, Eder Amayuelas ${ }^{\text {a }}$, Gotzone Barandika ${ }^{\text {cb* }}$, Edurne S. Larrea ${ }^{\text {a }}$, Begoña Bazán $^{\text {ab }}$, Miren Karmele Urtiaga ${ }^{\text {a }}$, Marta Iglesias ${ }^{d}$ and Maribel Arriortua $^{\text {ab }}$

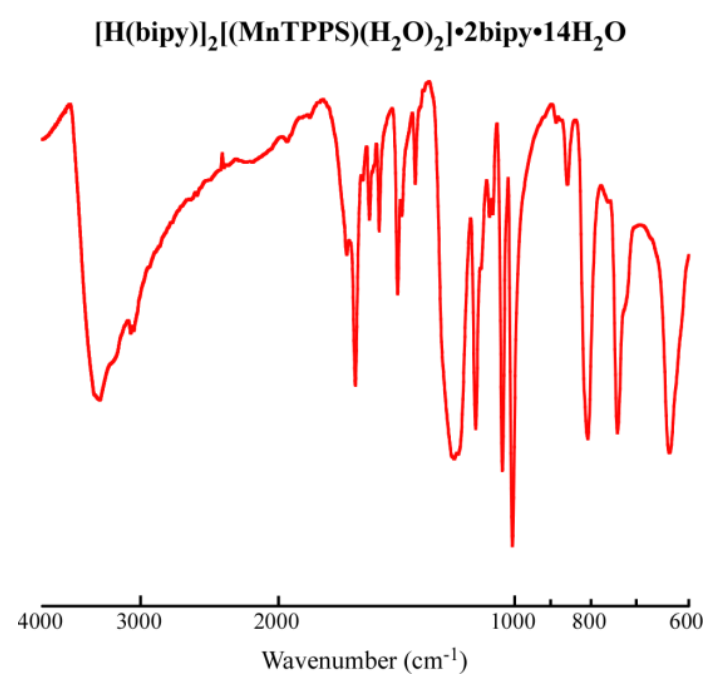

Figure S1 IR spectra for compound 1.
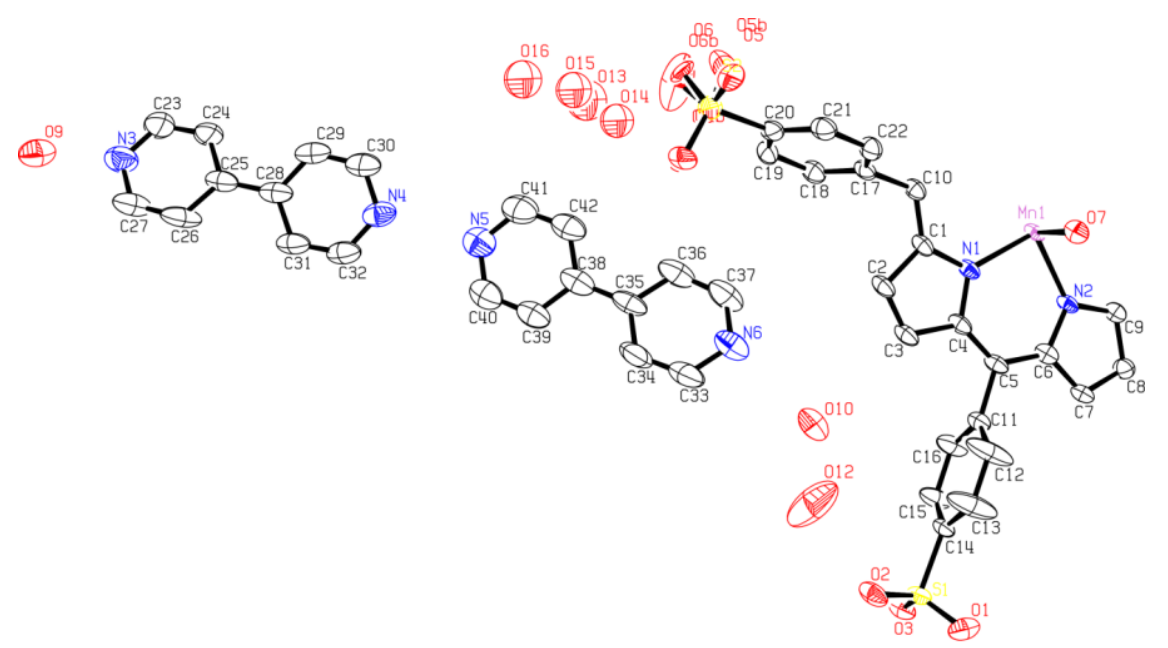

Figure S2 ORTEP detail for compound 1. The thermal ellipsoids correspond to $50 \%$ probability. Color code: $\mathrm{Mn}=\mathrm{Purple}, \mathrm{C}=$ black, $\mathrm{N}=$ blue, $\mathrm{O}=\mathrm{red}, \mathrm{S}=$ yellow. Hydrogen atoms have been omitted. 


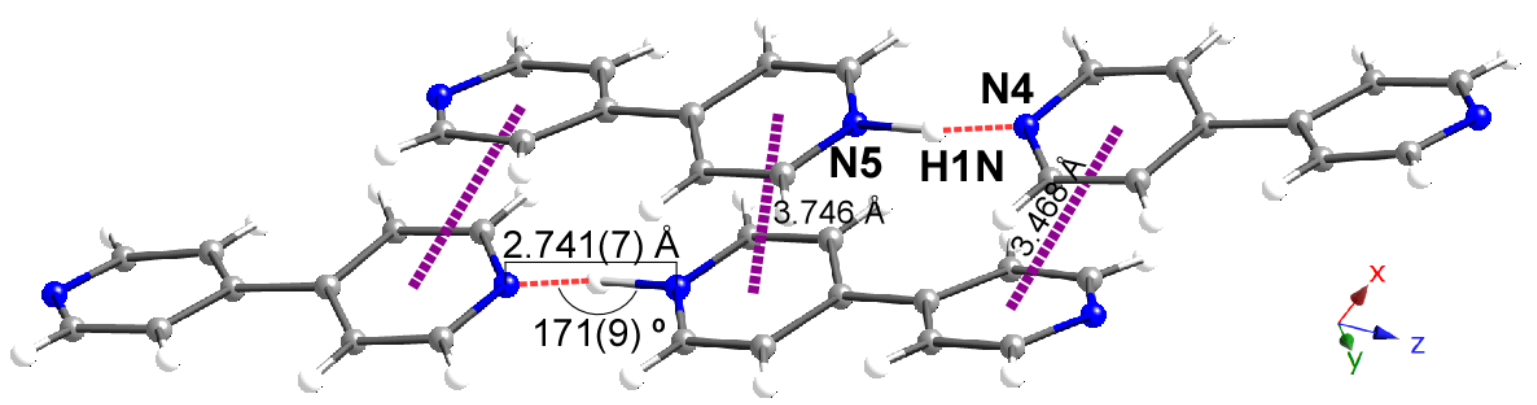

Figure S3 Detail of the $[\mathrm{H}(\text { bipy })]^{+}$cations for $[\mathrm{H}(\text { bipy })]_{2}\left[(\mathrm{MnTPPS})\left(\mathrm{H}_{2} \mathrm{O}\right)_{2}\right] \cdot 2$ bipy $\cdot 14 \mathrm{H}_{2} \mathrm{O}(\mathbf{1})$. Hydrogen bonds are marked in red dashed lines and $\pi-\pi$ interactions in purple dashed lines. Color code: $\mathrm{C}$, grey; $\mathrm{N}$, blue; and $\mathrm{H}$, white.

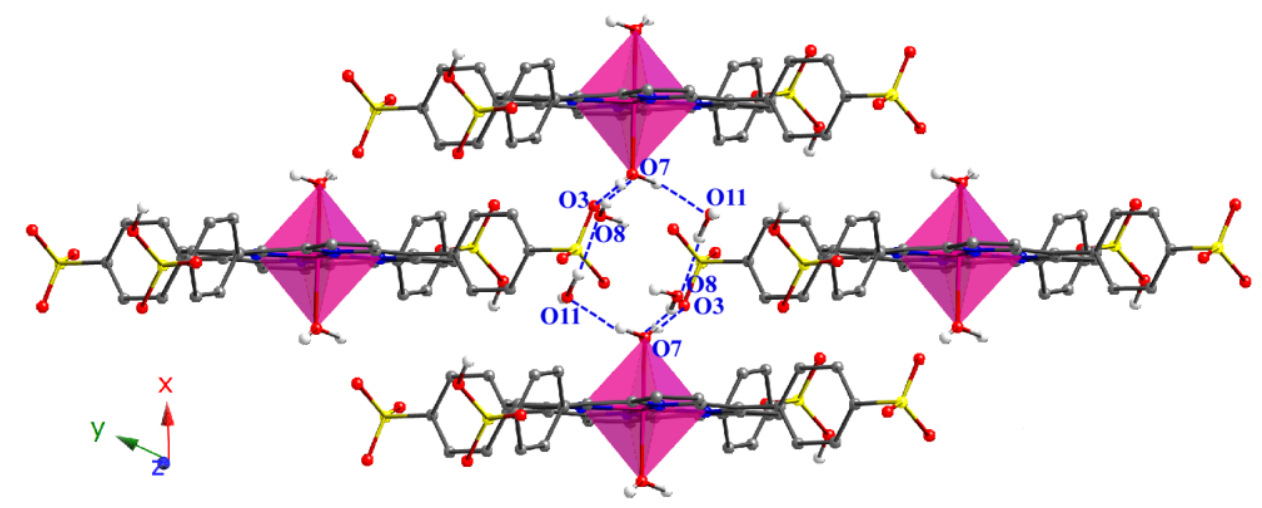

Figure S4 Detail for the H-bonding system in compound 1. Color code: Mn, pink; C, grey; N, blue; $\mathrm{O}$, red; $\mathrm{S}$, yellow and $\mathrm{H}$, white. Hydrogen bonds are marked as blue dashed lines. Porphyrin ring $\mathrm{H}$ atoms and $\mathrm{H}$ (bipy) cations have been omitted for clarity.

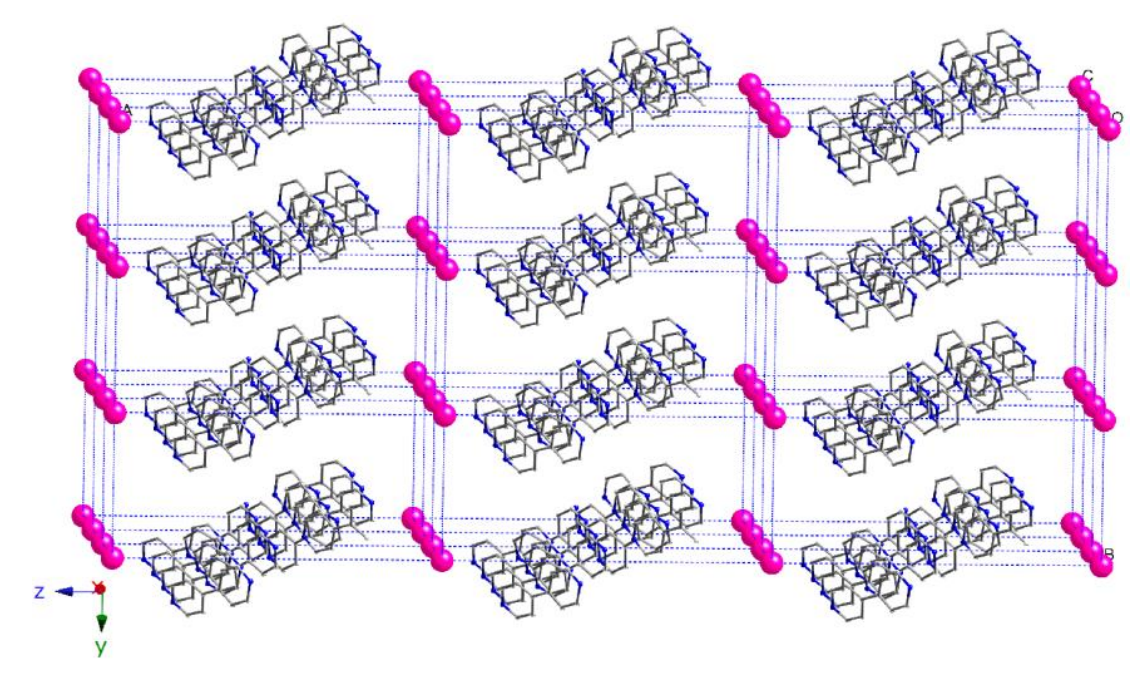

Figure S5 Simplification of the structure for compound 1 showing the relative position of the $[\mathrm{H}(\text { bipy })]^{+}$cations inside the Mn-node net. Color code: Mn, pink; C, grey and N, blue. 


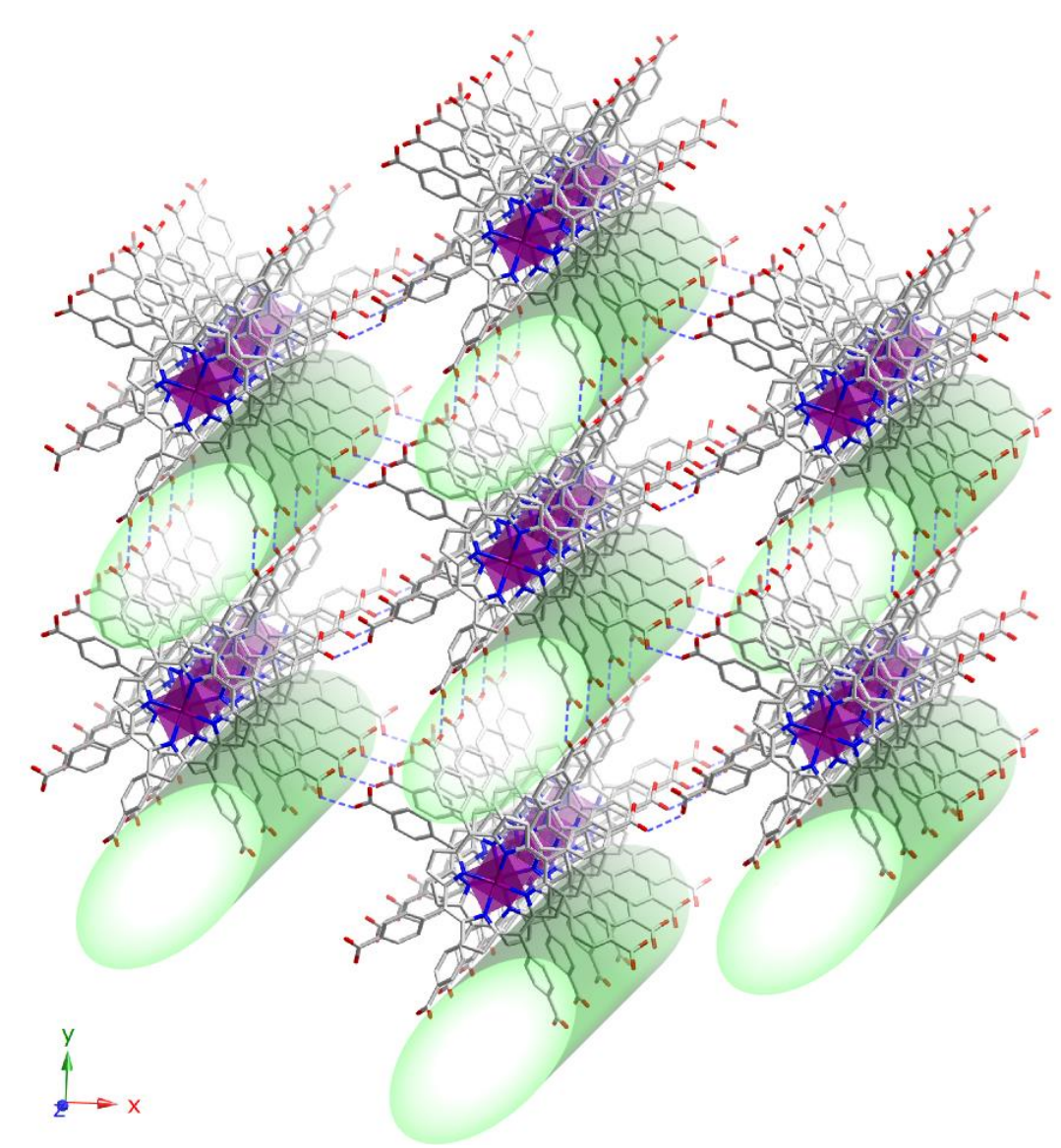

Figure S6 Perspective view of the stacked $x y \mathrm{H}$-bonded layers for compound $\mu-\mathrm{O}-[\mathrm{FeTCPP}]_{2} \cdot 16 \mathrm{DMF}$ (2). Accessible pathways are represented with green cylinders. Color code: Fe, purple; C, grey; N, blue and $\mathrm{O}$, red.

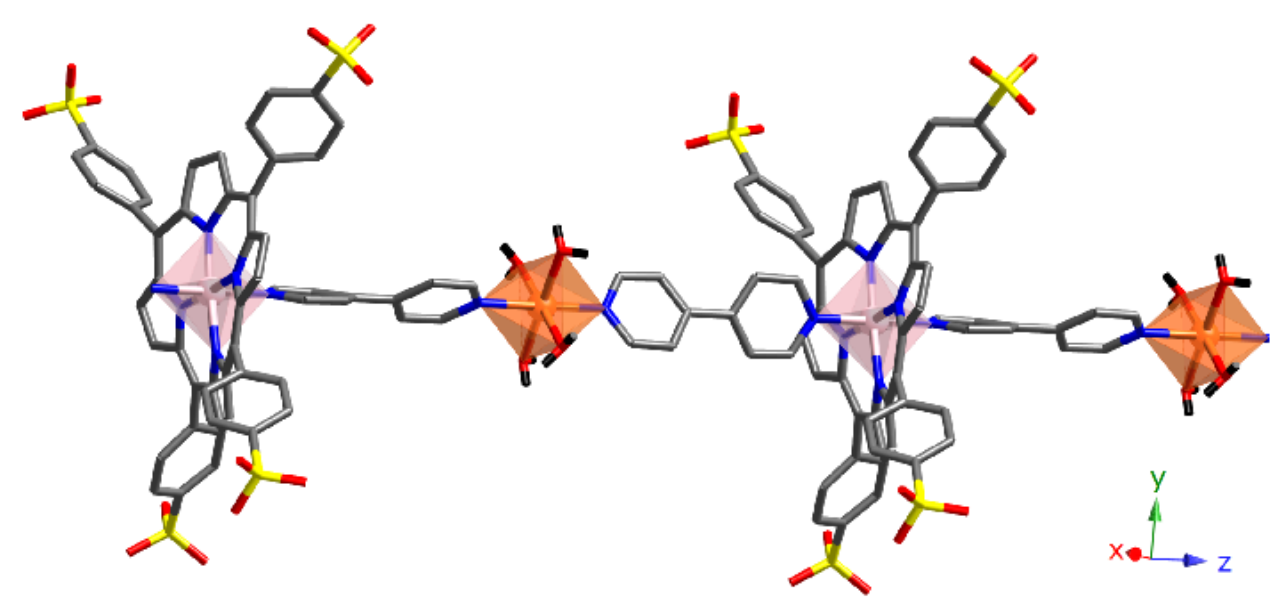

Figure S7 Detail of the structure for compound [CoTPPS $0.5($ bipy $\left.)\left(\mathrm{H}_{2} \mathrm{O}\right)_{2}\right] \cdot 6 \mathrm{H}_{2} \mathrm{O}$ (3) showing the extension of the 1D polymers. Color codes: $\mathrm{Co}(1)$ (TPPS) in pink, $\mathrm{Co}(2)$ in orange, $\mathrm{N}$ in blue, $\mathrm{C}$ in grey, $\mathrm{O}$ in red, $\mathrm{S}$ in yellow. $\mathrm{H}$ atoms (except those of water molecules, in black) have been omitted for clarity. 


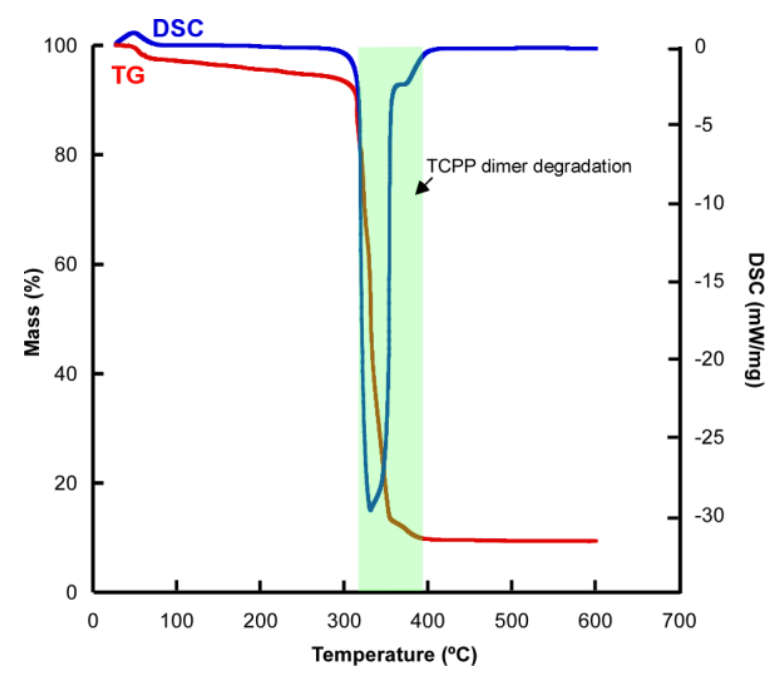

Figure S8 Thermogravimetric analysis (TG/DSC) for activated compound 2.
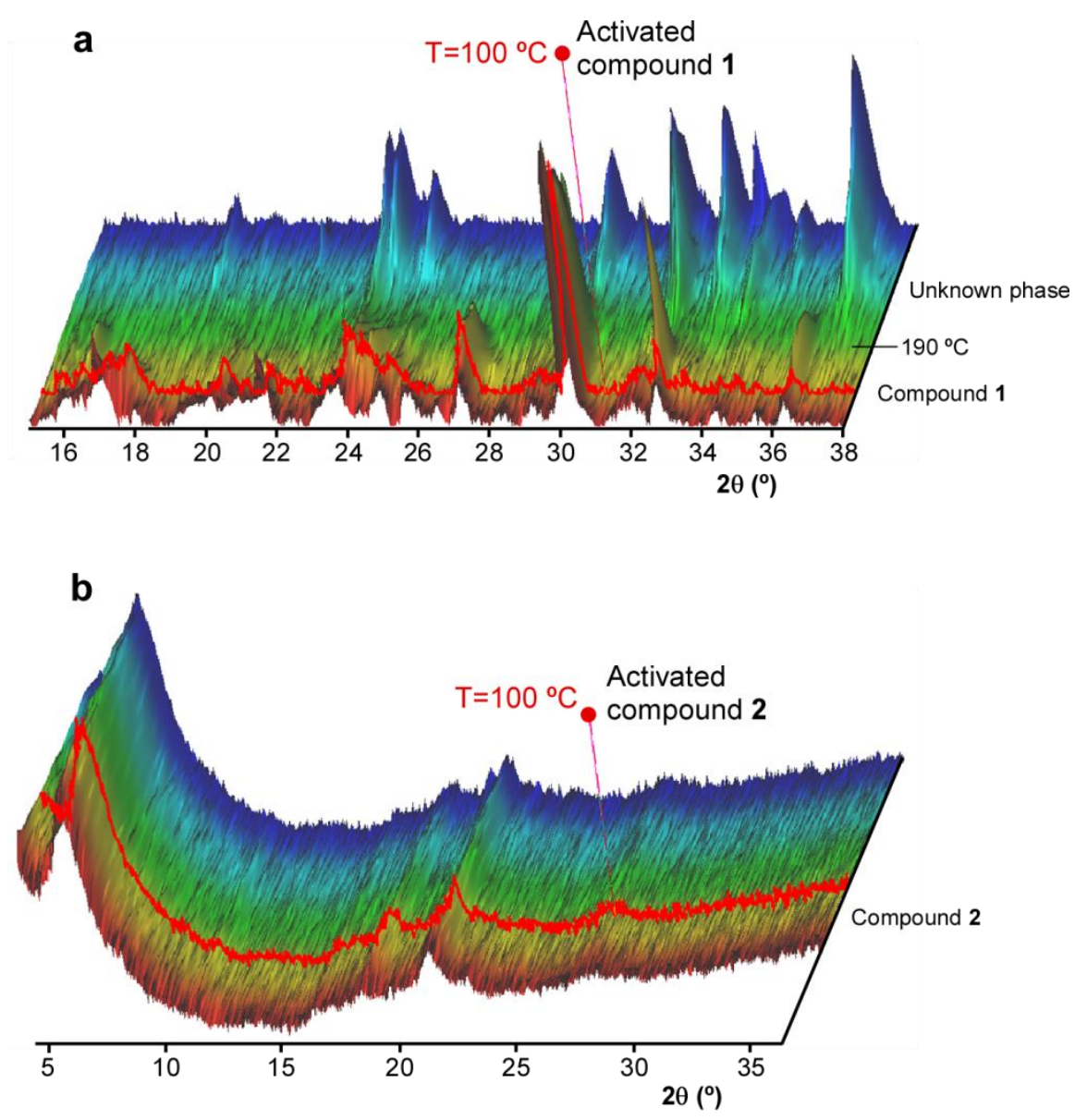

Figure S9 X-ray thermo-diffraction analysis for compounds $\mathbf{1}$ (a) and $\mathbf{2}$ (b). 


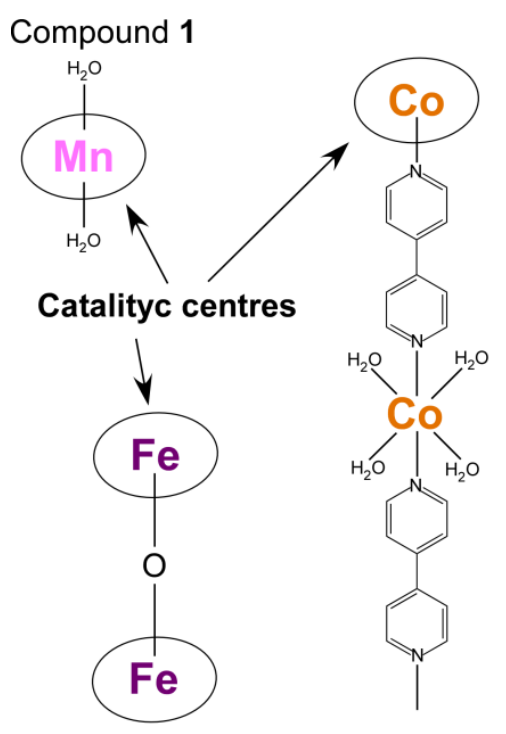

Compound 2 Compound $\mathbf{3}$

Figure S10Simplified scheme for compounds 1, 2 and 3. The porphyrin ring is represented with a black circle (TPPS for $\mathbf{1}$ and $\mathbf{3}$, and TCPP for $\mathbf{2}$ ).

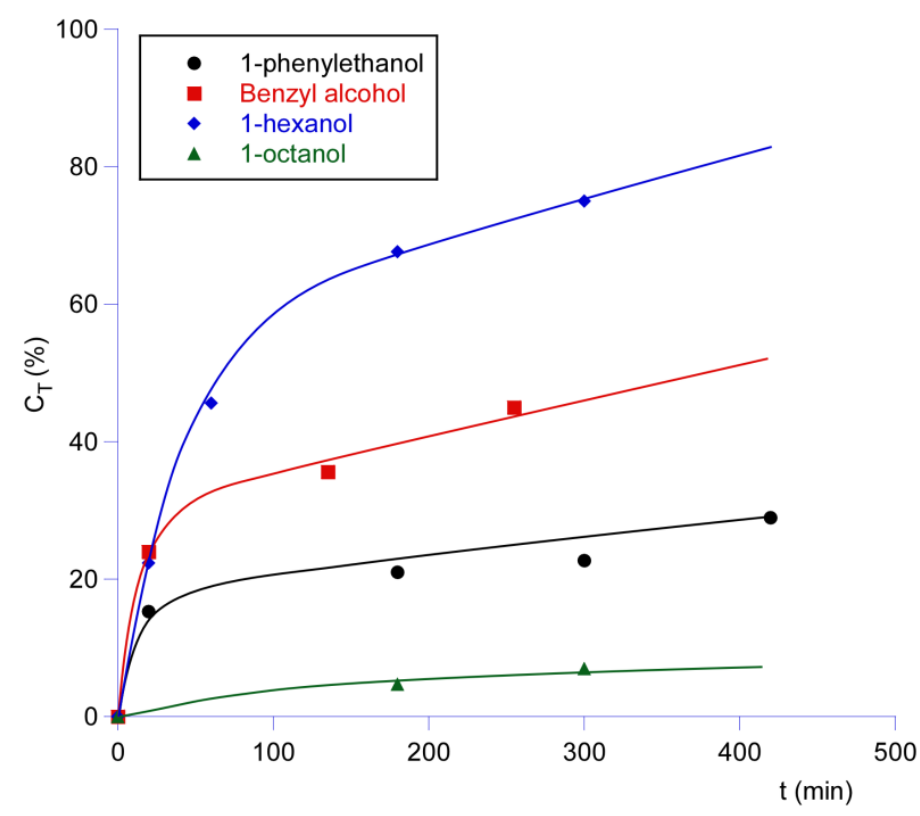

Figure S11Kinetic profiles for alcohols oxidation using compound $\mathbf{1}$ as catalyst. 


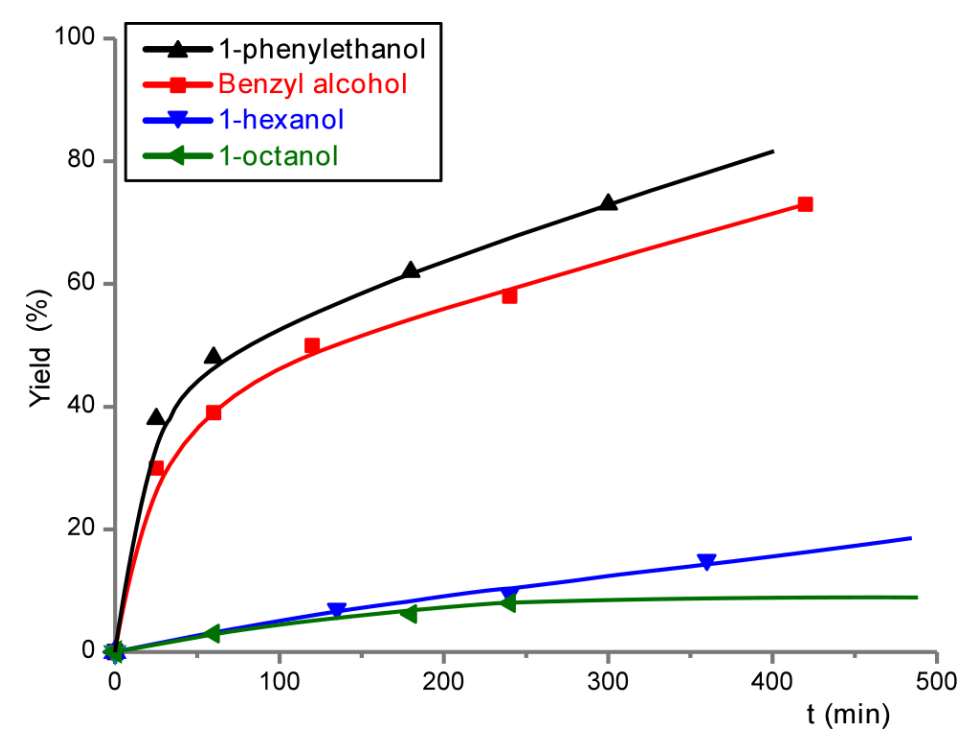

Figure S12 Kinetic profiles for alcohols oxidation using compound 2 as catalyst.

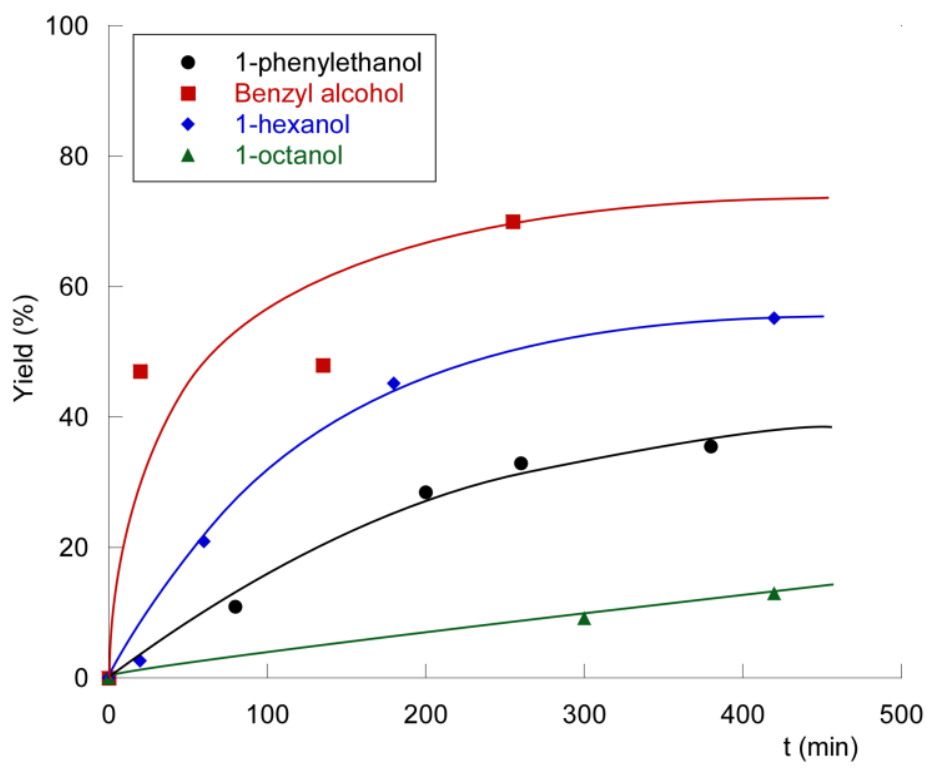

Figure S13 Kinetic profiles for alcohols oxidation using compound $\mathbf{3}$ as catalyst. 


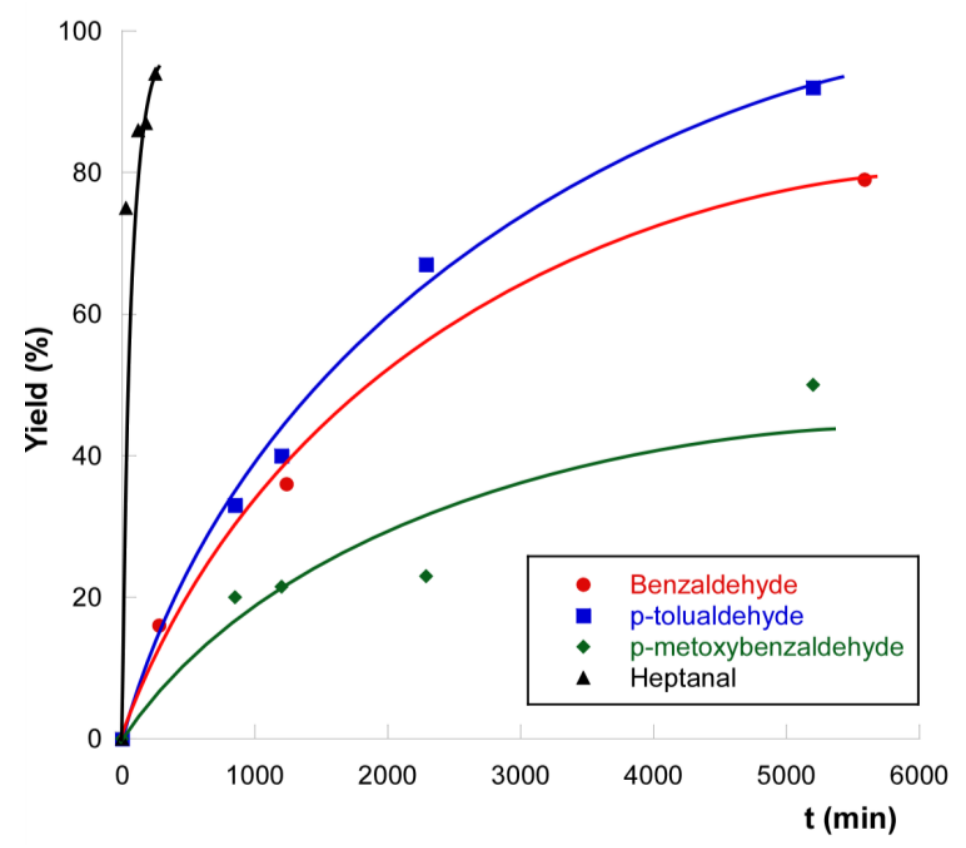

Figure S14 Kinetic profiles for the aldol condensation using compound $\mathbf{2}$ as catalyst.

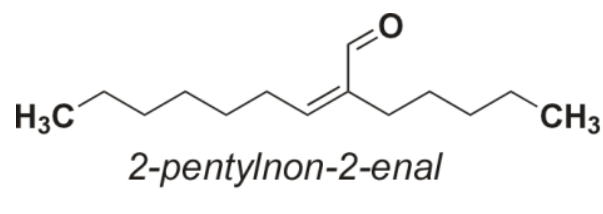

$\mathbf{a}$<smiles>CCCCC/C=C/CC(C)=O</smiles>

b<smiles>CCCCCC/C=C/C(C)=O</smiles>

c

Figure S15 Aldol condensation products for the heptanal substrate. 


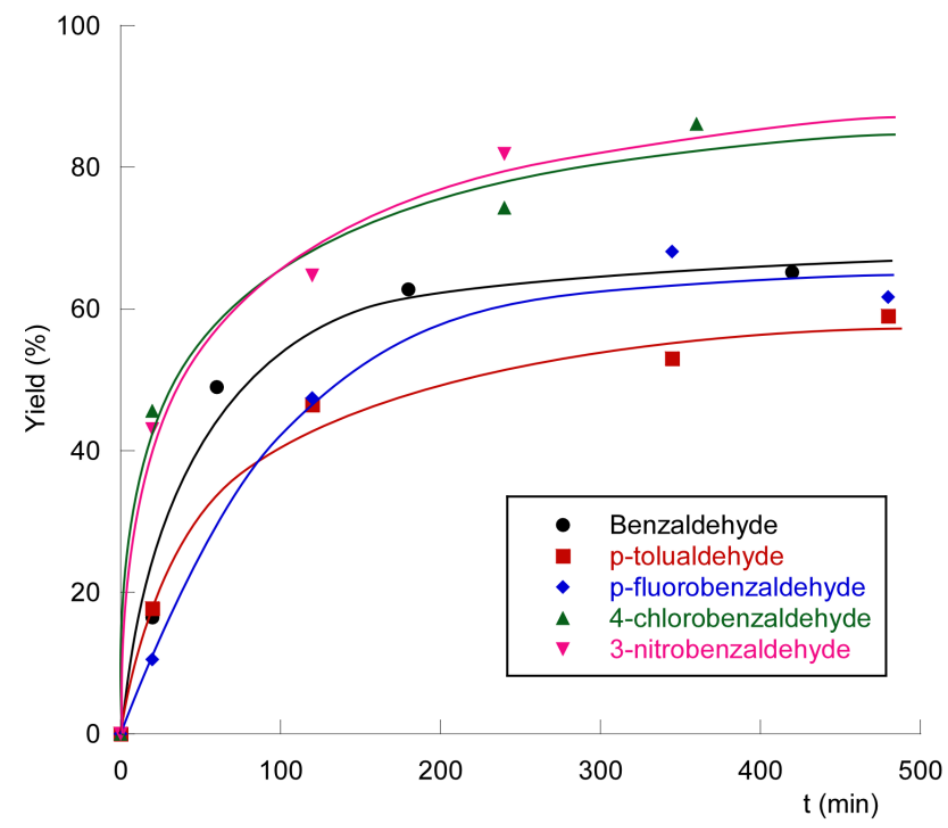

Figure S16 Kinetic profile for the Knoevenagel condensation using compound 2 as catalyst.

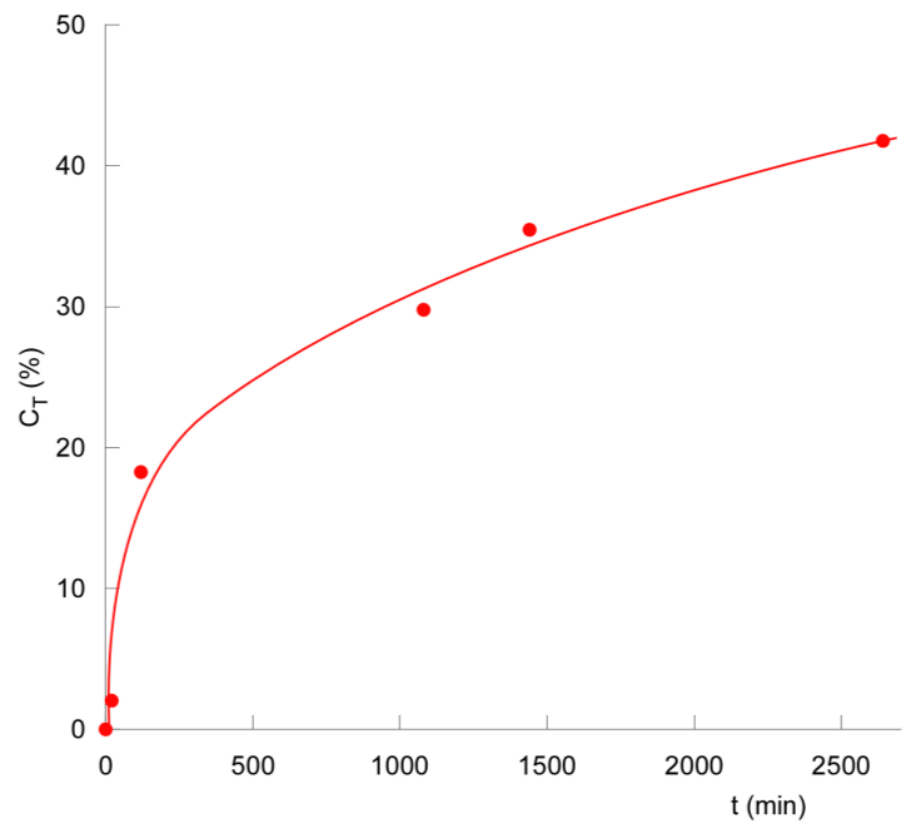

Figure S17 Kinetic profile for the acetal hydrolysis and Knoevenagel condensation one-pot cascade reaction using compound $\mathbf{2}$ as catalyst. 
a
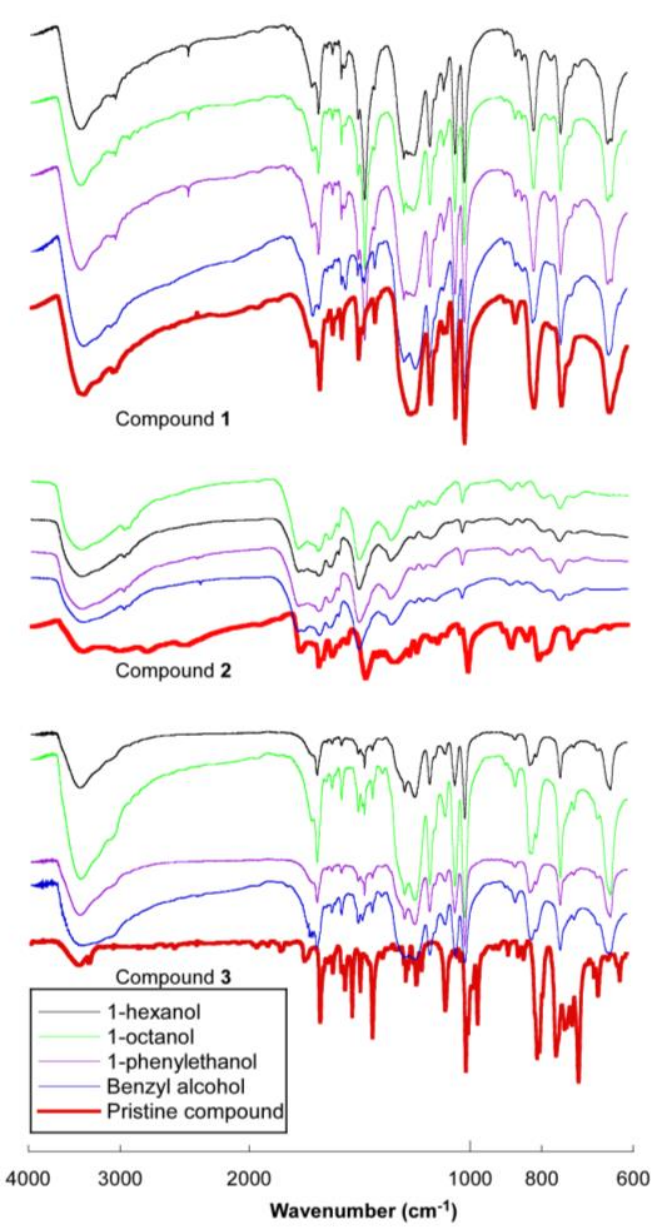

C
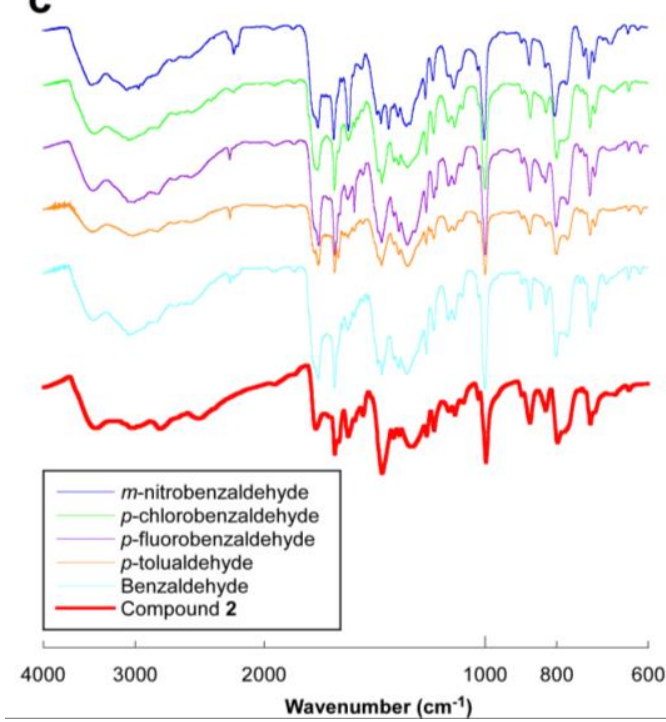

b

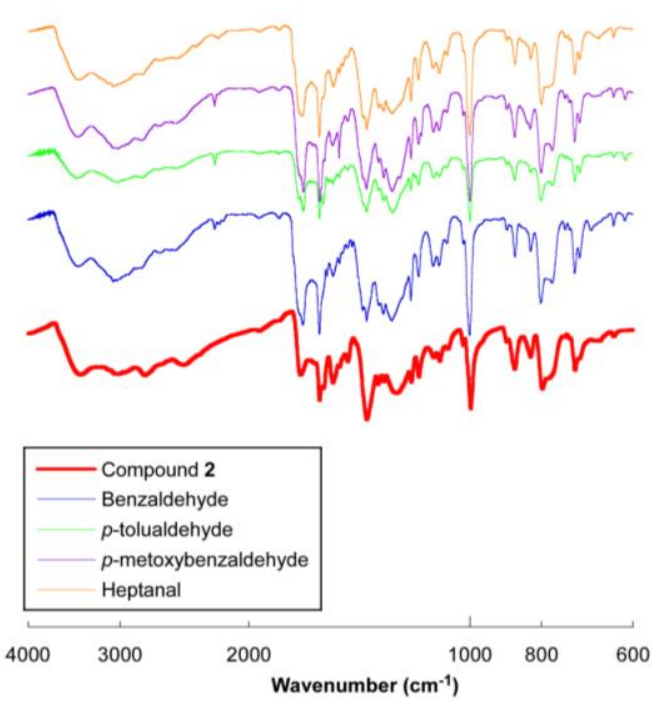

d

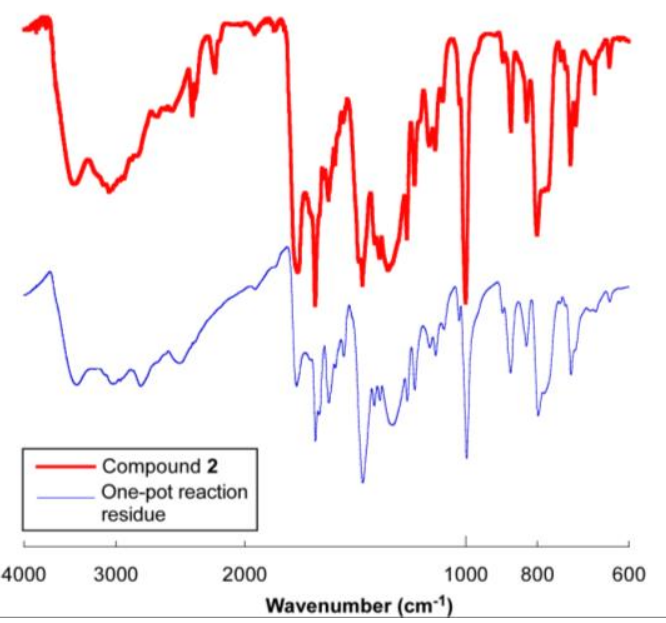

Figure S18 Infrared spectra for the recovered residues after oxidation reactions (a), aldol condensations (b), Knoevenagel condensations (c) and acetal hydrolysis and Knoevenagel condensation one-pot cascade reaction $(\mathrm{d})$. 

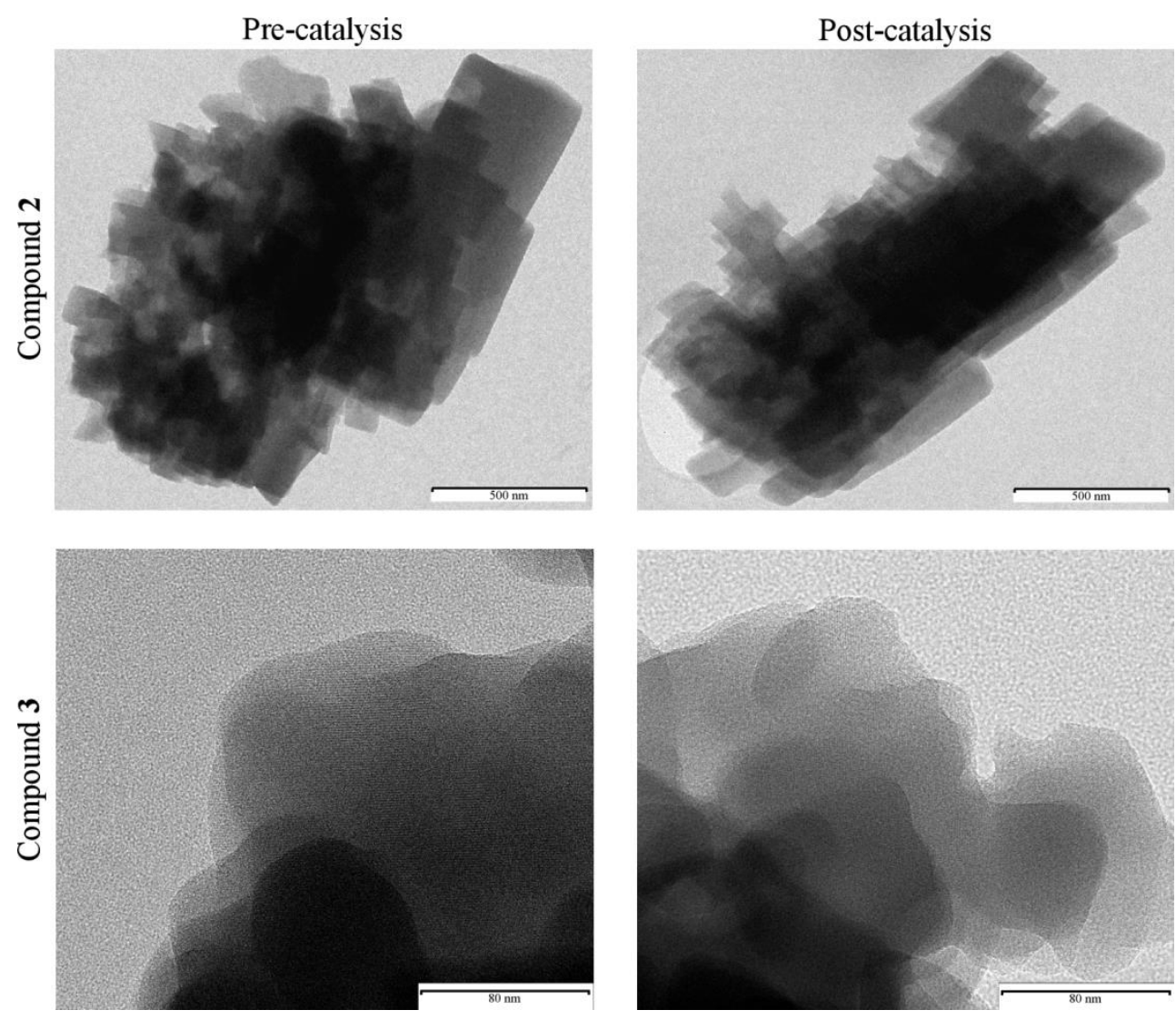

Figure S19 TEM particles morphology for compound $\mathbf{2}$ and $\mathbf{3}$ before and after the catalytic reactions. 
Table S1 Bond distances $(\AA)$ and angles $\left({ }^{\circ}\right)$ for compound 1.

\begin{tabular}{|c|c|c|c|}
\hline $\mathrm{C} 1-\mathrm{N} 1$ & $1.376(5)$ & $\mathrm{C} 33-\mathrm{C} 34$ & $1.374(9)$ \\
\hline $\mathrm{C} 1-\mathrm{C} 10$ & $1.397(5)$ & C33-H33 & 0.95 \\
\hline $\mathrm{C} 1-\mathrm{C} 2$ & $1.437(5)$ & $\mathrm{C} 34-\mathrm{C} 35$ & $1.377(8)$ \\
\hline $\mathrm{C} 2-\mathrm{C} 3$ & $1.350(6)$ & C34-H34 & 0.95 \\
\hline $\mathrm{C} 2-\mathrm{H} 2$ & 0.95 & $\mathrm{C} 35-\mathrm{C} 36$ & $1.397(7)$ \\
\hline $\mathrm{C} 3-\mathrm{C} 4$ & $1.436(5)$ & $\mathrm{C} 35-\mathrm{C} 38$ & $1.462(8)$ \\
\hline $\mathrm{C} 3-\mathrm{H} 3$ & 0.95 & $\mathrm{C} 36-\mathrm{C} 37$ & $1.374(9)$ \\
\hline $\mathrm{C} 4-\mathrm{N} 1$ & $1.380(5)$ & $\mathrm{C} 36-\mathrm{H} 36$ & 0.95 \\
\hline $\mathrm{C} 4-\mathrm{C} 5$ & $1.390(5)$ & $\mathrm{C} 37-\mathrm{N} 6$ & $1.328(7)$ \\
\hline $\mathrm{C} 5-\mathrm{C} 6$ & $1.392(5)$ & C37-H37 & 0.95 \\
\hline $\mathrm{C} 5-\mathrm{C} 11$ & $1.500(5)$ & C $38-\mathrm{C} 39$ & $1.393(7)$ \\
\hline $\mathrm{C} 6-\mathrm{N} 2$ & $1.383(5)$ & $\mathrm{C} 38-\mathrm{C} 42$ & $1.405(8)$ \\
\hline $\mathrm{C} 6-\mathrm{C} 7$ & $1.427(5)$ & $\mathrm{C} 39-\mathrm{C} 40$ & $1.357(8)$ \\
\hline $\mathrm{C} 7-\mathrm{C} 8$ & $1.345(5)$ & С $39-\mathrm{H} 39$ & 0.95 \\
\hline $\mathrm{C} 7-\mathrm{H} 7$ & 0.95 & $\mathrm{C} 40-\mathrm{N} 5$ & $1.346(7)$ \\
\hline $\mathrm{C} 8-\mathrm{C} 9$ & $1.437(5)$ & $\mathrm{C} 40-\mathrm{H} 40$ & 0.95 \\
\hline $\mathrm{C} 8-\mathrm{H} 8$ & 0.95 & $\mathrm{C} 41-\mathrm{N} 5$ & $1.338(8)$ \\
\hline $\mathrm{C} 9-\mathrm{N} 2$ & $1.381(5)$ & $\mathrm{C} 41-\mathrm{C} 42$ & $1.361(9)$ \\
\hline $\mathrm{C} 9-\mathrm{C} 10^{\mathrm{i}}$ & $1.393(5)$ & $\mathrm{C} 41-\mathrm{H} 41$ & 0.95 \\
\hline $\mathrm{C} 10-\mathrm{C} 9^{\mathrm{i}}$ & $1.393(5)$ & $\mathrm{C} 42-\mathrm{H} 42$ & 0.95 \\
\hline $\mathrm{C} 10-\mathrm{C} 17$ & $1.494(5)$ & $\mathrm{N} 1-\mathrm{Mn} 1$ & $2.012(3)$ \\
\hline $\mathrm{C} 11-\mathrm{C} 12$ & $1.365(6)$ & $\mathrm{N} 2-\mathrm{Mn} 1$ & $2.011(3)$ \\
\hline $\mathrm{C} 11-\mathrm{C} 16$ & $1.377(6)$ & $\mathrm{N} 5-\mathrm{H} 1 \mathrm{~N}$ & $1.28(9)$ \\
\hline $\mathrm{C} 12-\mathrm{C} 13$ & $1.381(7)$ & $\mathrm{O} 1-\mathrm{S} 1$ & $1.446(3)$ \\
\hline $\mathrm{C} 12-\mathrm{H} 12$ & 0.95 & $\mathrm{O} 2-\mathrm{S} 1$ & $1.451(3)$ \\
\hline $\mathrm{C} 13-\mathrm{C} 14$ & $1.377(6)$ & $\mathrm{O} 3-\mathrm{S} 1$ & $1.458(3)$ \\
\hline $\mathrm{C} 13-\mathrm{H} 13$ & 0.95 & $\mathrm{O} 4-\mathrm{S} 2$ & $1.42(4)$ \\
\hline $\mathrm{C} 14-\mathrm{C} 15$ & $1.356(6)$ & $\mathrm{O} 5-\mathrm{S} 2$ & $1.465(16)$ \\
\hline $\mathrm{C} 14-\mathrm{S} 1$ & $1.776(3)$ & $\mathrm{O} 6-\mathrm{S} 2$ & $1.425(15)$ \\
\hline $\mathrm{C} 15-\mathrm{C} 16$ & $1.384(6)$ & $\mathrm{O} 6-\mathrm{H} 6$ & $0.851(10)$ \\
\hline $\mathrm{C} 15-\mathrm{H} 15$ & 0.95 & O6- $-\mathrm{H} 6 \mathrm{~B}$ & $1.13(5)$ \\
\hline $\mathrm{C} 16-\mathrm{H} 16$ & 0.95 & $\mathrm{O} 4 \mathrm{~B}-\mathrm{S} 2$ & $1.46(4)$ \\
\hline $\mathrm{C} 17-\mathrm{C} 22$ & $1.388(5)$ & $\mathrm{O} 5 \mathrm{~B}-\mathrm{S} 2$ & $1.44(2)$ \\
\hline $\mathrm{C} 17-\mathrm{C} 18$ & $1.391(6)$ & $\mathrm{O} 6 \mathrm{~B}-\mathrm{S} 2$ & $1.41(2)$ \\
\hline $\mathrm{C} 18-\mathrm{C} 19$ & $1.390(6)$ & $\mathrm{O} 6 \mathrm{~B}-\mathrm{H} 6$ & $0.62(4)$ \\
\hline $\mathrm{C} 18-\mathrm{H} 18$ & 0.95 & $\mathrm{O} 6 \mathrm{~B}-\mathrm{H} 6 \mathrm{~B}$ & $0.851(10)$ \\
\hline $\mathrm{C} 19-\mathrm{C} 20$ & $1.379(6)$ & $\mathrm{O} 7-\mathrm{Mn} 1$ & $2.245(3)$ \\
\hline
\end{tabular}




\begin{tabular}{|c|c|c|c|}
\hline C19-H19 & 0.95 & $\mathrm{O} 7-\mathrm{H} 7 \mathrm{~A}$ & 0.8208 \\
\hline $\mathrm{C} 20-\mathrm{C} 21$ & $1.384(6)$ & $\mathrm{O} 7-\mathrm{H} 7 \mathrm{~B}$ & 0.8182 \\
\hline $\mathrm{C} 20-\mathrm{S} 2$ & $1.785(4)$ & $\mathrm{Mn} 1-\mathrm{N} 2^{\mathrm{i}}$ & $2.011(3)$ \\
\hline $\mathrm{C} 21-\mathrm{C} 22$ & $1.391(5)$ & $\mathrm{Mn} 1-\mathrm{N} 1^{\mathrm{i}}$ & $2.012(3)$ \\
\hline $\mathrm{C} 21-\mathrm{H} 21$ & 0.95 & $\mathrm{Mn} 1-\mathrm{O}^{\mathrm{i}}$ & $2.245(3)$ \\
\hline $\mathrm{C} 22-\mathrm{H} 22$ & 0.95 & $\mathrm{O} 8-\mathrm{H} 8 \mathrm{~A}$ & 0.8226 \\
\hline $\mathrm{C} 23-\mathrm{N} 3$ & $1.321(8)$ & $\mathrm{O} 8-\mathrm{H} 8 \mathrm{~B}$ & 0.8258 \\
\hline $\mathrm{C} 23-\mathrm{C} 24$ & $1.373(11)$ & $\mathrm{O} 9-\mathrm{H} 9 \mathrm{~A}$ & 0.8296 \\
\hline $\mathrm{C} 23-\mathrm{H} 23$ & 0.95 & $\mathrm{O} 9-\mathrm{H} 9 \mathrm{~B}$ & 0.8297 \\
\hline $\mathrm{C} 24-\mathrm{C} 25$ & $1.382(8)$ & $\mathrm{O} 10-\mathrm{H} 10 \mathrm{~A}$ & 0.8263 \\
\hline $\mathrm{C} 24-\mathrm{H} 24$ & 0.95 & $\mathrm{O} 10-\mathrm{H} 10 \mathrm{~B}$ & 0.8274 \\
\hline $\mathrm{C} 25-\mathrm{C} 26$ & $1.390(8)$ & $\mathrm{O} 11-\mathrm{H} 11 \mathrm{~A}$ & 0.8201 \\
\hline $\mathrm{C} 25-\mathrm{C} 28$ & $1.476(8)$ & $\mathrm{O} 11-\mathrm{H} 11 \mathrm{~B}$ & 0.8214 \\
\hline $\mathrm{C} 26-\mathrm{C} 27$ & $1.356(9)$ & $\mathrm{O} 12-\mathrm{H} 12 \mathrm{~B}$ & 0.8219 \\
\hline $\mathrm{C} 26-\mathrm{H} 26$ & 0.95 & $\mathrm{O} 12-\mathrm{H} 12 \mathrm{~A}$ & 0.8233 \\
\hline $\mathrm{C} 27-\mathrm{N} 3$ & $1.318(7)$ & $\mathrm{O} 13-\mathrm{H} 13 \mathrm{~B}$ & 0.8213 \\
\hline $\mathrm{C} 27-\mathrm{H} 27$ & 0.95 & $\mathrm{O} 13-\mathrm{H} 13 \mathrm{~A}$ & 0.8256 \\
\hline $\mathrm{C} 28-\mathrm{C} 29$ & $1.394(7)$ & $\mathrm{O} 13-\mathrm{H} 14 \mathrm{~A}$ & 1.2319 \\
\hline $\mathrm{C} 28-\mathrm{C} 31$ & $1.400(6)$ & O13-H16B & 1.441 \\
\hline $\mathrm{C} 29-\mathrm{C} 30$ & $1.361(8)$ & $\mathrm{O} 15-\mathrm{H} 15 \mathrm{~A}$ & 0.8237 \\
\hline $\mathrm{C} 29-\mathrm{H} 29$ & 0.95 & $\mathrm{O} 15-\mathrm{H} 15 \mathrm{~B}$ & 0.8244 \\
\hline $\mathrm{C} 30-\mathrm{N} 4$ & $1.345(7)$ & $\mathrm{O} 14-\mathrm{H} 13 \mathrm{~A}$ & 1.0074 \\
\hline $\mathrm{C} 30-\mathrm{H} 30$ & 0.95 & $\mathrm{O} 14-\mathrm{H} 14 \mathrm{~A}$ & 0.8156 \\
\hline $\mathrm{C} 31-\mathrm{C} 32$ & $1.350(8)$ & $\mathrm{O} 14-\mathrm{H} 14 \mathrm{~B}$ & 0.8249 \\
\hline $\mathrm{C} 31-\mathrm{H} 31$ & 0.95 & O16-H13B & 1.2329 \\
\hline $\mathrm{C} 32-\mathrm{N} 4$ & $1.342(7)$ & $\mathrm{O} 16-\mathrm{H} 16 \mathrm{~A}$ & 0.8327 \\
\hline $\mathrm{C} 32-\mathrm{H} 32$ & 0.95 & O16-H16B & 0.8243 \\
\hline $\mathrm{C} 33-\mathrm{N} 6$ & $1.342(7)$ & & \\
\hline \multicolumn{2}{|l|}{ Angles } & $\mathrm{N} 6-\mathrm{C} 33-\mathrm{H} 33$ & 118.3 \\
\hline $\mathrm{N} 1-\mathrm{C} 1-\mathrm{C} 10$ & $126.0(3)$ & $\mathrm{C} 34-\mathrm{C} 33-\mathrm{H} 33$ & 118.3 \\
\hline $\mathrm{N} 1-\mathrm{C} 1-\mathrm{C} 2$ & $109.6(3)$ & $\mathrm{C} 33-\mathrm{C} 34-\mathrm{C} 35$ & $120.7(5)$ \\
\hline $\mathrm{C} 10-\mathrm{C} 1-\mathrm{C} 2$ & $124.3(3)$ & $\mathrm{C} 33-\mathrm{C} 34-\mathrm{H} 34$ & 119.6 \\
\hline $\mathrm{C} 3-\mathrm{C} 2-\mathrm{C} 1$ & $107.2(3)$ & $\mathrm{C} 35-\mathrm{C} 34-\mathrm{H} 34$ & 119.6 \\
\hline $\mathrm{C} 3-\mathrm{C} 2-\mathrm{H} 2$ & 126.4 & $\mathrm{C} 34-\mathrm{C} 35-\mathrm{C} 36$ & $115.5(6)$ \\
\hline $\mathrm{C} 1-\mathrm{C} 2-\mathrm{H} 2$ & 126.4 & $\mathrm{C} 34-\mathrm{C} 35-\mathrm{C} 38$ & $122.2(5)$ \\
\hline $\mathrm{C} 2-\mathrm{C} 3-\mathrm{C} 4$ & $107.5(3)$ & $\mathrm{C} 36-\mathrm{C} 35-\mathrm{C} 38$ & $122.3(5)$ \\
\hline $\mathrm{C} 2-\mathrm{C} 3-\mathrm{H} 3$ & 126.3 & $\mathrm{C} 37-\mathrm{C} 36-\mathrm{C} 35$ & $120.6(5)$ \\
\hline $\mathrm{C} 4-\mathrm{C} 3-\mathrm{H} 3$ & 126.3 & $\mathrm{C} 37-\mathrm{C} 36-\mathrm{H} 36$ & 119.7 \\
\hline $\mathrm{N} 1-\mathrm{C} 4-\mathrm{C} 5$ & $126.3(3)$ & $\mathrm{C} 35-\mathrm{C} 36-\mathrm{H} 36$ & 119.7 \\
\hline
\end{tabular}




\begin{tabular}{|c|c|c|c|}
\hline $\mathrm{N} 1-\mathrm{C} 4-\mathrm{C} 3$ & $109.4(3)$ & N6-C37-C36 & $123.4(5)$ \\
\hline $\mathrm{C} 5-\mathrm{C} 4-\mathrm{C} 3$ & $124.4(3)$ & N6-C37-H37 & 118.3 \\
\hline $\mathrm{C} 4-\mathrm{C} 5-\mathrm{C} 6$ & $124.6(3)$ & $\mathrm{C} 36-\mathrm{C} 37-\mathrm{H} 37$ & 118.3 \\
\hline $\mathrm{C} 4-\mathrm{C} 5-\mathrm{C} 11$ & $117.6(3)$ & $\mathrm{C} 39-\mathrm{C} 38-\mathrm{C} 42$ & $115.8(6)$ \\
\hline $\mathrm{C} 6-\mathrm{C} 5-\mathrm{C} 11$ & $117.9(3)$ & $\mathrm{C} 39-\mathrm{C} 38-\mathrm{C} 35$ & $121.5(5)$ \\
\hline $\mathrm{N} 2-\mathrm{C} 6-\mathrm{C} 5$ & $125.4(3)$ & $\mathrm{C} 42-\mathrm{C} 38-\mathrm{C} 35$ & $122.7(5)$ \\
\hline $\mathrm{N} 2-\mathrm{C} 6-\mathrm{C} 7$ & $109.7(3)$ & $\mathrm{C} 40-\mathrm{C} 39-\mathrm{C} 38$ & $120.9(5)$ \\
\hline $\mathrm{C} 5-\mathrm{C} 6-\mathrm{C} 7$ & $124.8(3)$ & $\mathrm{C} 40-\mathrm{C} 39-\mathrm{H} 39$ & 119.5 \\
\hline $\mathrm{C} 8-\mathrm{C} 7-\mathrm{C} 6$ & $107.5(3)$ & $\mathrm{C} 38-\mathrm{C} 39-\mathrm{H} 39$ & 119.5 \\
\hline $\mathrm{C} 8-\mathrm{C} 7-\mathrm{H} 7$ & 126.3 & $\mathrm{~N} 5-\mathrm{C} 40-\mathrm{C} 39$ & $121.9(6)$ \\
\hline $\mathrm{C} 6-\mathrm{C} 7-\mathrm{H} 7$ & 126.3 & $\mathrm{~N} 5-\mathrm{C} 40-\mathrm{H} 40$ & 119 \\
\hline $\mathrm{C} 7-\mathrm{C} 8-\mathrm{C} 9$ & $107.6(3)$ & $\mathrm{C} 39-\mathrm{C} 40-\mathrm{H} 40$ & 119 \\
\hline $\mathrm{C} 7-\mathrm{C} 8-\mathrm{H} 8$ & 126.2 & $\mathrm{~N} 5-\mathrm{C} 41-\mathrm{C} 42$ & $121.8(6)$ \\
\hline $\mathrm{C} 9-\mathrm{C} 8-\mathrm{H} 8$ & 126.2 & $\mathrm{~N} 5-\mathrm{C} 41-\mathrm{H} 41$ & 119.1 \\
\hline $\mathrm{N} 2-\mathrm{C} 9-\mathrm{C} 10^{\mathrm{i}}$ & $126.4(3)$ & $\mathrm{C} 42-\mathrm{C} 41-\mathrm{H} 41$ & 119.1 \\
\hline $\mathrm{N} 2-\mathrm{C} 9-\mathrm{C} 8$ & $109.2(3)$ & $\mathrm{C} 41-\mathrm{C} 42-\mathrm{C} 38$ & $120.8(6)$ \\
\hline $\mathrm{C} 10^{\mathrm{i}}-\mathrm{C} 9-\mathrm{C} 8$ & $124.4(3)$ & $\mathrm{C} 41-\mathrm{C} 42-\mathrm{H} 42$ & 119.6 \\
\hline $\mathrm{C} 9^{\mathrm{i}}-\mathrm{C} 10-\mathrm{C} 1$ & $123.2(3)$ & $\mathrm{C} 38-\mathrm{C} 42-\mathrm{H} 42$ & 119.6 \\
\hline $\mathrm{C} 9^{\mathrm{i}}-\mathrm{C} 10-\mathrm{C} 17$ & $117.8(3)$ & $\mathrm{C} 1-\mathrm{N} 1-\mathrm{C} 4$ & $106.3(3)$ \\
\hline $\mathrm{C} 1-\mathrm{C} 10-\mathrm{C} 17$ & $119.0(3)$ & $\mathrm{C} 1-\mathrm{N} 1-\mathrm{Mn} 1$ & $127.4(2)$ \\
\hline $\mathrm{C} 12-\mathrm{C} 11-\mathrm{C} 16$ & $118.4(4)$ & $\mathrm{C} 4-\mathrm{N} 1-\mathrm{Mn} 1$ & $126.3(2)$ \\
\hline $\mathrm{C} 12-\mathrm{C} 11-\mathrm{C} 5$ & $121.4(4)$ & $\mathrm{C} 9-\mathrm{N} 2-\mathrm{C} 6$ & $106.0(3)$ \\
\hline $\mathrm{C} 16-\mathrm{C} 11-\mathrm{C} 5$ & $120.2(4)$ & $\mathrm{C} 9-\mathrm{N} 2-\mathrm{Mn} 1$ & $127.1(2)$ \\
\hline $\mathrm{C} 11-\mathrm{C} 12-\mathrm{C} 13$ & $121.1(4)$ & $\mathrm{C} 6-\mathrm{N} 2-\mathrm{Mn} 1$ & $126.9(2)$ \\
\hline $\mathrm{C} 11-\mathrm{C} 12-\mathrm{H} 12$ & 119.5 & $\mathrm{C} 27-\mathrm{N} 3-\mathrm{C} 23$ & $117.8(6)$ \\
\hline $\mathrm{C} 13-\mathrm{C} 12-\mathrm{H} 12$ & 119.5 & $\mathrm{C} 32-\mathrm{N} 4-\mathrm{C} 30$ & $117.6(5)$ \\
\hline $\mathrm{C} 14-\mathrm{C} 13-\mathrm{C} 12$ & $120.1(4)$ & $\mathrm{C} 32-\mathrm{N} 4-\mathrm{H} 1 \mathrm{~N}$ & $120(4)$ \\
\hline $\mathrm{C} 14-\mathrm{C} 13-\mathrm{H} 13$ & 120 & $\mathrm{C} 30-\mathrm{N} 4-\mathrm{H} 1 \mathrm{~N}$ & $122(4)$ \\
\hline $\mathrm{C} 12-\mathrm{C} 13-\mathrm{H} 13$ & 120 & $\mathrm{C} 41-\mathrm{N} 5-\mathrm{C} 40$ & $118.8(6)$ \\
\hline $\mathrm{C} 15-\mathrm{C} 14-\mathrm{C} 13$ & $119.2(4)$ & $\mathrm{C} 41-\mathrm{N} 5-\mathrm{H} 1 \mathrm{~N}$ & $123(4)$ \\
\hline $\mathrm{C} 15-\mathrm{C} 14-\mathrm{S} 1$ & $121.2(3)$ & $\mathrm{C} 40-\mathrm{N} 5-\mathrm{H} 1 \mathrm{~N}$ & $118(4)$ \\
\hline $\mathrm{C} 13-\mathrm{C} 14-\mathrm{S} 1$ & $119.6(3)$ & $\mathrm{C} 37-\mathrm{N} 6-\mathrm{C} 33$ & $116.3(6)$ \\
\hline $\mathrm{C} 14-\mathrm{C} 15-\mathrm{C} 16$ & $120.7(4)$ & $\mathrm{S} 2-\mathrm{O} 6-\mathrm{H} 6$ & $115(3)$ \\
\hline $\mathrm{C} 14-\mathrm{C} 15-\mathrm{H} 15$ & 119.7 & $\mathrm{~S} 2-\mathrm{O} 6-\mathrm{H} 6 \mathrm{~B}$ & $98(2)$ \\
\hline $\mathrm{C} 16-\mathrm{C} 15-\mathrm{H} 15$ & 119.7 & $\mathrm{~S} 2-\mathrm{O} 6 \mathrm{~B}-\mathrm{H} 6$ & $142(5)$ \\
\hline $\mathrm{C} 11-\mathrm{C} 16-\mathrm{C} 15$ & $120.5(4)$ & $\mathrm{S} 2-\mathrm{O} 6 \mathrm{~B}-\mathrm{H} 6 \mathrm{~B}$ & $116(3)$ \\
\hline $\mathrm{C} 11-\mathrm{C} 16-\mathrm{H} 16$ & 119.7 & $\mathrm{Mn} 1-\mathrm{O} 7-\mathrm{H} 7 \mathrm{~A}$ & 116.4 \\
\hline $\mathrm{C} 15-\mathrm{C} 16-\mathrm{H} 16$ & 119.7 & $\mathrm{Mn} 1-\mathrm{O} 7-\mathrm{H} 7 \mathrm{~B}$ & 109.2 \\
\hline $\mathrm{C} 22-\mathrm{C} 17-\mathrm{C} 18$ & $119.2(3)$ & $\mathrm{H} 7 \mathrm{~A}-\mathrm{O} 7-\mathrm{H} 7 \mathrm{~B}$ & 110.6 \\
\hline
\end{tabular}




\begin{tabular}{|c|c|c|c|}
\hline $\mathrm{C} 22-\mathrm{C} 17-\mathrm{C} 10$ & $118.9(3)$ & $\mathrm{O} 1-\mathrm{S} 1-\mathrm{O} 2$ & $111.6(2)$ \\
\hline $\mathrm{C} 18-\mathrm{C} 17-\mathrm{C} 10$ & $121.9(3)$ & $\mathrm{O} 1-\mathrm{S} 1-\mathrm{O} 3$ & $113.68(18)$ \\
\hline $\mathrm{C} 19-\mathrm{C} 18-\mathrm{C} 17$ & $120.3(4)$ & $\mathrm{O} 2-\mathrm{S} 1-\mathrm{O} 3$ & $112.49(18)$ \\
\hline $\mathrm{C} 19-\mathrm{C} 18-\mathrm{H} 18$ & 119.9 & $\mathrm{O} 1-\mathrm{S} 1-\mathrm{C} 14$ & 106.19 (19) \\
\hline $\mathrm{C} 17-\mathrm{C} 18-\mathrm{H} 18$ & 119.9 & $\mathrm{O} 2-\mathrm{S} 1-\mathrm{C} 14$ & $106.04(17)$ \\
\hline $\mathrm{C} 20-\mathrm{C} 19-\mathrm{C} 18$ & $119.8(4)$ & $\mathrm{O} 3-\mathrm{S} 1-\mathrm{C} 14$ & $106.21(17)$ \\
\hline $\mathrm{C} 20-\mathrm{C} 19-\mathrm{H} 19$ & 120.1 & $\mathrm{O} 6 \mathrm{~B}-\mathrm{S} 2-\mathrm{O} 4$ & $101.7(19)$ \\
\hline $\mathrm{C} 18-\mathrm{C} 19-\mathrm{H} 19$ & 120.1 & $\mathrm{O} 4-\mathrm{S} 2-\mathrm{O} 6$ & $112.7(18)$ \\
\hline $\mathrm{C} 19-\mathrm{C} 20-\mathrm{C} 21$ & $120.8(4)$ & $\mathrm{O} 6 \mathrm{~B}-\mathrm{S} 2-\mathrm{O} 5 \mathrm{~B}$ & $111.0(14)$ \\
\hline $\mathrm{C} 19-\mathrm{C} 20-\mathrm{S} 2$ & $119.6(3)$ & $\mathrm{O} 4-\mathrm{S} 2-\mathrm{O} 5 \mathrm{~B}$ & $123.1(16)$ \\
\hline $\mathrm{C} 21-\mathrm{C} 20-\mathrm{S} 2$ & $119.6(3)$ & $\mathrm{O} 6-\mathrm{S} 2-\mathrm{O} 5 \mathrm{~B}$ & $100.0(12)$ \\
\hline $\mathrm{C} 20-\mathrm{C} 21-\mathrm{C} 22$ & $119.2(4)$ & $\mathrm{O} 6 \mathrm{~B}-\mathrm{S} 2-\mathrm{O} 4 \mathrm{~B}$ & $113(2)$ \\
\hline $\mathrm{C} 20-\mathrm{C} 21-\mathrm{H} 21$ & 120.4 & $\mathrm{O} 6-\mathrm{S} 2-\mathrm{O} 4 \mathrm{~B}$ & $122.5(18)$ \\
\hline $\mathrm{C} 22-\mathrm{C} 21-\mathrm{H} 21$ & 120.4 & $\mathrm{O} 5 \mathrm{~B}-\mathrm{S} 2-\mathrm{O} 4 \mathrm{~B}$ & $109.6(16)$ \\
\hline $\mathrm{C} 17-\mathrm{C} 22-\mathrm{C} 21$ & $120.8(4)$ & $\mathrm{O} 6 \mathrm{~B}-\mathrm{S} 2-\mathrm{O} 5$ & $123.4(12)$ \\
\hline $\mathrm{C} 17-\mathrm{C} 22-\mathrm{H} 22$ & 119.6 & $\mathrm{O} 4-\mathrm{S} 2-\mathrm{O} 5$ & $115.9(15)$ \\
\hline $\mathrm{C} 21-\mathrm{C} 22-\mathrm{H} 22$ & 119.6 & $\mathrm{O} 6-\mathrm{S} 2-\mathrm{O} 5$ & $112.4(10)$ \\
\hline $\mathrm{N} 3-\mathrm{C} 23-\mathrm{C} 24$ & $122.1(6)$ & $\mathrm{O} 4 \mathrm{~B}-\mathrm{S} 2-\mathrm{O} 5$ & $102.0(15)$ \\
\hline $\mathrm{N} 3-\mathrm{C} 23-\mathrm{H} 23$ & 118.9 & $\mathrm{O} 6 \mathrm{~B}-\mathrm{S} 2-\mathrm{C} 20$ & $106.1(8)$ \\
\hline $\mathrm{C} 24-\mathrm{C} 23-\mathrm{H} 23$ & 118.9 & $\mathrm{O} 4-\mathrm{S} 2-\mathrm{C} 20$ & $103.7(16)$ \\
\hline $\mathrm{C} 23-\mathrm{C} 24-\mathrm{C} 25$ & $121.6(6)$ & $\mathrm{O} 6-\mathrm{S} 2-\mathrm{C} 20$ & $106.7(6)$ \\
\hline $\mathrm{C} 23-\mathrm{C} 24-\mathrm{H} 24$ & 119.2 & $\mathrm{O} 5 \mathrm{~B}-\mathrm{S} 2-\mathrm{C} 20$ & $109.8(8)$ \\
\hline $\mathrm{C} 25-\mathrm{C} 24-\mathrm{H} 24$ & 119.2 & $\mathrm{O} 4 \mathrm{~B}-\mathrm{S} 2-\mathrm{C} 20$ & $107.6(16)$ \\
\hline $\mathrm{C} 24-\mathrm{C} 25-\mathrm{C} 26$ & $114.0(6)$ & $\mathrm{O} 5-\mathrm{S} 2-\mathrm{C} 20$ & $104.2(6)$ \\
\hline $\mathrm{C} 24-\mathrm{C} 25-\mathrm{C} 28$ & $122.3(5)$ & $\mathrm{N} 2-\mathrm{Mn} 1-\mathrm{N} 2^{\mathrm{i}}$ & $180.00(9)$ \\
\hline $\mathrm{C} 26-\mathrm{C} 25-\mathrm{C} 28$ & $123.6(4)$ & $\mathrm{N} 2-\mathrm{Mn} 1-\mathrm{N} 1$ & $90.43(12)$ \\
\hline $\mathrm{C} 27-\mathrm{C} 26-\mathrm{C} 25$ & $121.6(5)$ & $\mathrm{N} 2 \mathrm{i}-\mathrm{Mn} 1-\mathrm{N} 1$ & $89.57(12)$ \\
\hline $\mathrm{C} 27-\mathrm{C} 26-\mathrm{H} 26$ & 119.2 & $\mathrm{~N} 2-\mathrm{Mn} 1-\mathrm{N} 1^{\mathrm{i}}$ & $89.57(12)$ \\
\hline $\mathrm{C} 25-\mathrm{C} 26-\mathrm{H} 26$ & 119.2 & $\mathrm{~N} 2{ }^{\mathrm{i}}-\mathrm{Mn} 1-\mathrm{N} 1^{\mathrm{i}}$ & $90.43(12)$ \\
\hline $\mathrm{N} 3-\mathrm{C} 27-\mathrm{C} 26$ & $122.8(6)$ & $\mathrm{N} 1-\mathrm{Mn} 1-\mathrm{N} 1^{\mathrm{i}}$ & $180.00(18)$ \\
\hline $\mathrm{N} 3-\mathrm{C} 27-\mathrm{H} 27$ & 118.6 & $\mathrm{~N} 2-\mathrm{Mn} 1-\mathrm{O} 7$ & $90.10(11)$ \\
\hline $\mathrm{C} 26-\mathrm{C} 27-\mathrm{H} 27$ & 118.6 & $\mathrm{~N} 2 \mathrm{i}-\mathrm{Mn} 1-\mathrm{O} 7$ & $89.90(11)$ \\
\hline $\mathrm{C} 29-\mathrm{C} 28-\mathrm{C} 31$ & $116.3(5)$ & $\mathrm{N} 1-\mathrm{Mn} 1-\mathrm{O} 7$ & $90.98(11)$ \\
\hline $\mathrm{C} 29-\mathrm{C} 28-\mathrm{C} 25$ & $121.4(4)$ & $\mathrm{N} 1{ }^{\mathrm{i}}-\mathrm{Mn} 1-\mathrm{O} 7$ & $89.02(11)$ \\
\hline $\mathrm{C} 31-\mathrm{C} 28-\mathrm{C} 25$ & $122.4(5)$ & $\mathrm{N} 2-\mathrm{Mn} 1-\mathrm{O}^{\mathrm{i}}$ & $89.90(11)$ \\
\hline $\mathrm{C} 30-\mathrm{C} 29-\mathrm{C} 28$ & $120.1(5)$ & $\mathrm{N} 2{ }^{\mathrm{i}}-\mathrm{Mn} 1-\mathrm{O}^{\mathrm{i}}$ & $90.10(11)$ \\
\hline $\mathrm{C} 30-\mathrm{C} 29-\mathrm{H} 29$ & 120 & $\mathrm{~N} 1-\mathrm{Mn} 1-\mathrm{O} 7^{\mathrm{i}}$ & $89.02(11)$ \\
\hline $\mathrm{C} 28-\mathrm{C} 29-\mathrm{H} 29$ & 120 & $\mathrm{~N} 1{ }^{\mathrm{i}}-\mathrm{Mn} 1-\mathrm{O}^{\mathrm{i}}$ & $90.98(11)$ \\
\hline $\mathrm{N} 4-\mathrm{C} 30-\mathrm{C} 29$ & $122.8(6)$ & $\mathrm{O} 7-\mathrm{Mn} 1-\mathrm{O} 7^{\mathrm{i}}$ & 180 \\
\hline
\end{tabular}




\begin{tabular}{l|l|l|l}
\hline $\mathrm{N} 4-\mathrm{C} 30-\mathrm{H} 30$ & 118.6 & $\mathrm{H} 8 \mathrm{~A}-\mathrm{O} 8-\mathrm{H} 8 \mathrm{~B}$ & 108.2 \\
\hline $\mathrm{C} 29-\mathrm{C} 30-\mathrm{H} 30$ & 118.6 & $\mathrm{H} 9 \mathrm{~A}-\mathrm{O} 9-\mathrm{H} 9 \mathrm{~B}$ & 107.5 \\
\hline $\mathrm{C} 32-\mathrm{C} 31-\mathrm{C} 28$ & $120.5(5)$ & $\mathrm{H} 10 \mathrm{~A}-\mathrm{O} 10-\mathrm{H} 10 \mathrm{~B}$ & 108.9 \\
\hline $\mathrm{C} 32-\mathrm{C} 31-\mathrm{H} 31$ & 119.7 & $\mathrm{H} 11 \mathrm{~A}-\mathrm{O} 11-\mathrm{H} 11 \mathrm{~B}$ & 111 \\
\hline $\mathrm{C} 28-\mathrm{C} 31-\mathrm{H} 31$ & 119.7 & $\mathrm{H} 12 \mathrm{~B}-\mathrm{O} 12-\mathrm{H} 12 \mathrm{~A}$ & 109.8 \\
\hline $\mathrm{N} 4-\mathrm{C} 32-\mathrm{C} 31$ & $122.7(5)$ & $\mathrm{H} 14 \mathrm{~A}-\mathrm{O} 13-\mathrm{H} 16 \mathrm{~B}$ & 96.2 \\
\hline $\mathrm{N} 4-\mathrm{C} 32-\mathrm{H} 32$ & 118.6 & $\mathrm{H} 14 \mathrm{~A}-\mathrm{O} 14-\mathrm{H} 14 \mathrm{~B}$ & 112.4 \\
\hline $\mathrm{C} 31-\mathrm{C} 32-\mathrm{H} 32$ & 118.6 & $\mathrm{H} 16 \mathrm{~A}-\mathrm{O} 16-\mathrm{H} 16 \mathrm{~B}$ & 108.4 \\
\hline $\mathrm{N} 6-\mathrm{C} 33-\mathrm{C} 34$ & $123.5(6)$ & & \\
\hline $\mathrm{S} y m m$ & &
\end{tabular}

Symmetry code: (i) $-x+2,-y,-z+2$.

Table S2 Fractional atomic coordinates and isotropic or equivalent isotropic displacement parameters $\left(\AA^{2}\right)$ for compound $\mathbf{1}$.

\begin{tabular}{|c|c|c|c|c|c|}
\hline Atoms & $x$ & $y$ & $z$ & $U_{\text {iso }} * / U_{\text {eq }}$ & Occ. $(<1)$ \\
\hline C1 & $0.9495(4)$ & $0.0089(4)$ & $0.8643(2)$ & $0.0273(8)$ & \\
\hline $\mathrm{C2}$ & 0.8818 & $0.1008(4)$ & $0.8172(2)$ & $0.0316(8)$ & \\
\hline H2 & 0.8754 & 0.0795 & 0.7762 & $0.038 *$ & \\
\hline $\mathrm{C3}$ & $0.8290(4)$ & $0.2230(4)$ & $0.8420(2)$ & $0.0324(8)$ & \\
\hline H3 & 0.7784 & 0.3037 & 0.8217 & $0.039 *$ & \\
\hline C4 & $0.8633(4)$ & $0.2087(4)$ & $0.9048(2)$ & $0.0273(8)$ & \\
\hline C5 & $0.8294(4)$ & $0.3134(4)$ & $0.9451(2)$ & $0.0274(8)$ & \\
\hline C6 & $0.8582(4)$ & $0.3003(4)$ & $1.0065(2)$ & $0.0274(7)$ & \\
\hline C7 & $0.8193(4)$ & $0.4074(4)$ & $1.0485(2)$ & $0.0305(8)$ & \\
\hline H7 & 0.7759 & 0.4984 & 1.039 & $0.037 *$ & \\
\hline C8 & $0.8555(5)$ & $0.3562(4)$ & $1.1041(2)$ & $0.0306(8)$ & \\
\hline H8 & 0.8408 & 0.4044 & 1.141 & $0.037^{*}$ & \\
\hline C9 & $0.9204(4)$ & $0.2152(3)$ & $1.0972(2)$ & $0.0256(7)$ & \\
\hline C10 & $1.0239(4)$ & -0.1277 (4) & $0.8554(2)$ & $0.0273(8)$ & \\
\hline C11 & $0.7547(4)$ & $0.4501(3)$ & $0.9207(2)$ & $0.0282(8)$ & \\
\hline $\mathrm{C} 12$ & $0.8397(6)$ & $0.5117(6)$ & $0.8944(3)$ & $0.079(2)$ & \\
\hline H12 & 0.9484 & 0.4683 & 0.8926 & $0.094 *$ & \\
\hline $\mathrm{C} 13$ & $0.7704(6)$ & $0.6359(6)$ & $0.8703(4)$ & $0.083(2)$ & \\
\hline H13 & 0.8314 & 0.6771 & 0.852 & $0.099 *$ & \\
\hline C14 & $0.6130(4)$ & $0.7001(3)$ & $0.8728(2)$ & $0.0264(7)$ & \\
\hline C15 & $0.5276(5)$ & $0.6409(4)$ & $0.9002(2)$ & $0.045(1)$ & \\
\hline H15 & 0.4188 & 0.6856 & 0.9032 & $0.053^{*}$ & \\
\hline C16 & $0.5973(5)$ & $0.5159(4)$ & $0.9239(2)$ & $0.044(1)$ & \\
\hline
\end{tabular}




\begin{tabular}{|c|c|c|c|c|c|}
\hline H16 & 0.5361 & 0.4752 & 0.9425 & $0.053^{*}$ & \\
\hline $\mathrm{C17}$ & $1.0508(4)$ & $-0.1838(3)$ & $0.7917(2)$ & $0.0279(8)$ & \\
\hline $\mathrm{C} 18$ & $0.9326(5)$ & -0.1859 (4) & $0.7619(2)$ & $0.0338(9)$ & \\
\hline H18 & 0.8309 & -0.1498 & 0.7818 & $0.041 *$ & \\
\hline C19 & $0.9626(5)$ & $-0.2407(4)$ & $0.7030(2)$ & 0.039 (1) & \\
\hline H19 & 0.8815 & -0.2419 & 0.6826 & $0.047^{*}$ & \\
\hline $\mathrm{C2O}$ & $1.1103(5)$ & $-0.2934(4)$ & $0.6742(2)$ & $0.0361(9)$ & \\
\hline $\mathrm{C} 21$ & $1.2292(5)$ & -0.2910 & $0.7029(2)$ & $0.0373(9)$ & \\
\hline H21 & 1.3304 & -0.326 & 0.6826 & $0.045^{*}$ & \\
\hline $\mathrm{C22}$ & $1.1987(5)$ & $-0.2366(4)$ & $0.7611(2)$ & 0.0337 (9) & \\
\hline H22 & 1.28 & -0.2357 & 0.7822 & $0.04 *$ & \\
\hline $\mathrm{C23}$ & $-0.176(1)$ & $-0.4032(7)$ & $0.2379(3)$ & $0.114(4)$ & \\
\hline $\mathrm{H} 23$ & -0.1813 & -0.4854 & 0.2407 & $0.137^{*}$ & \\
\hline $\mathrm{C} 24$ & $-0.100(1)$ & $-0.3722(6)$ & $0.2801(3)$ & $0.098(3)$ & \\
\hline H24 & -0.0515 & -0.4351 & 0.3099 & $0.118^{*}$ & \\
\hline $\mathrm{C25}$ & $-0.0941(6)$ & $-0.2517(4)$ & $0.2801(2)$ & $0.048(1)$ & \\
\hline $\mathrm{C} 26$ & $-0.1697(6)$ & $-0.1681(5)$ & $0.2344(3)$ & $0.068(2)$ & \\
\hline H26 & -0.1754 & -0.0816 & 0.2327 & $0.082^{*}$ & \\
\hline $\mathrm{C} 27$ & $-0.2355(6)$ & $-0.2069(5)$ & $0.1923(3)$ & $0.065(2)$ & \\
\hline H27 & -0.2802 & -0.1484 & 0.1603 & $0.078^{*}$ & \\
\hline $\mathrm{C28}$ & $-0.0207(6)$ & $-0.2142(4)$ & $0.3270(2)$ & 0.047 (1) & \\
\hline $\mathrm{C29}$ & $0.0774(6)$ & $-0.3079(5)$ & $0.3644(3)$ & $0.059(1)$ & \\
\hline H29 & 0.0998 & -0.3982 & 0.3595 & $0.071^{*}$ & \\
\hline C30 & $0.1410(7)$ & $-0.2700(5)$ & $0.4081(3)$ & 0.064 (1) & \\
\hline H30 & 0.2063 & -0.3353 & 0.4335 & $0.077^{*}$ & \\
\hline C31 & $-0.0454(7)$ & $-0.0836(4)$ & $0.3366(2)$ & $0.056(1)$ & \\
\hline H31 & -0.1103 & -0.016 & 0.312 & $0.067 *$ & \\
\hline $\mathrm{C} 32$ & $0.0224(7)$ & $-0.0532(5)$ & $0.3805(2)$ & $0.059(2)$ & \\
\hline H32 & 0.0034 & 0.0363 & 0.386 & $0.07 *$ & \\
\hline $\mathrm{C33}$ & $0.4985(7)$ & $0.2878(6)$ & $0.6435(3)$ & $0.069(2)$ & \\
\hline H33 & 0.4908 & 0.3737 & 0.6354 & $0.084^{*}$ & \\
\hline C34 & $0.4365(7)$ & $0.2345(5)$ & $0.6048(3)$ & $0.069(2)$ & \\
\hline H34 & 0.3868 & 0.2843 & 0.5713 & $0.083^{*}$ & \\
\hline C35 & $0.4457(5)$ & 0.1098 (5) & $0.6140(2)$ & 0.049 (1) & \\
\hline C36 & $0.5189(7)$ & $0.0452(6)$ & 0.6649 (3) & $0.066(2)$ & \\
\hline H36 & 0.5298 & -0.0414 & 0.6736 & $0.079 *$ & \\
\hline C37 & $0.5753(7)$ & $0.1059(6)$ & $0.7024(3)$ & $0.070(2)$ & \\
\hline H37 & 0.6209 & 0.0603 & 0.7374 & $0.084 *$ & \\
\hline
\end{tabular}




\begin{tabular}{|c|c|c|c|c|c|}
\hline $\mathrm{C38}$ & $0.3791(5)$ & $0.0507(5)$ & $0.5743(2)$ & $0.052(1)$ & \\
\hline C39 & $0.2766(6)$ & $0.1258(5)$ & $0.5332(2)$ & $0.056(1)$ & \\
\hline H39 & 0.2497 & 0.2175 & 0.5304 & $0.067^{*}$ & \\
\hline C40 & $0.2146(7)$ & $0.0699(6)$ & $0.4970(3)$ & $0.061(1)$ & \\
\hline H40 & 0.1454 & 0.1235 & 0.4692 & $0.073^{*}$ & \\
\hline C41 & $0.3478(7)$ & $-0.1349(6)$ & $0.5378(3)$ & $0.070(2)$ & \\
\hline H41 & 0.373 & -0.2264 & 0.5393 & $0.084 *$ & \\
\hline $\mathrm{C} 42$ & $0.4138(6)$ & $-0.0842(5)$ & $0.5750(3)$ & $0.062(1)$ & \\
\hline H42 & 0.4841 & -0.1406 & 0.6017 & $0.074 *$ & \\
\hline N1 & $0.9354(3)$ & $0.0765(3)$ & 0.9179 (1) & $0.0255(6)$ & \\
\hline N2 & $0.9225(3)$ & $0.1823(3)$ & 1.0367 (1) & $0.0245(6)$ & \\
\hline N3 & $-0.2400(6)$ & $-0.3224(4)$ & $0.1937(2)$ & $0.0650(1)$ & \\
\hline N4 & $0.1154(5)$ & $-0.1440(4)$ & $0.4167(2)$ & $0.056(1)$ & \\
\hline N5 & $0.2484(5)$ & $-0.0594(5)$ & $0.4993(2)$ & $0.0601(1)$ & \\
\hline N6 & $0.5694(5)$ & $0.2248(5)$ & $0.6921(2)$ & $0.0619(1)$ & \\
\hline 01 & $0.5987(4)$ & $0.9340(3)$ & $0.8599(2)$ & $0.0468(8)$ & \\
\hline $\mathbf{0 2}$ & $0.5578(4)$ & $0.8331(3)$ & $0.7729(1)$ & $0.0438(8)$ & \\
\hline $\mathbf{O 3}$ & $0.3623(3)$ & $0.9093(3)$ & 0.8595 (1) & $0.0353(6)$ & \\
\hline O4 & $1.073(4)$ & $-0.243(4)$ & $0.5621(2)$ & $0.043(6)$ & 0.5 \\
\hline 05 & $1.314(2)$ & $-0.423(2)$ & $0.5860(7)$ & $0.046(3)$ & 0.5 \\
\hline O6 & $1.079(2)$ & $-0.447(1)$ & $0.5964(6)$ & $0.057(4)$ & 0.5 \\
\hline O4B & $1.109(4)$ & $-0.245(4)$ & $0.5559(2)$ & $0.042(6)$ & 0.5 \\
\hline O5B & $1.306(3)$ & $-0.447(2)$ & $0.5844(9)$ & $0.117(1)$ & 0.5 \\
\hline O6B & $1.052(3)$ & $-0.422(2)$ & $0.5937(9)$ & $0.111(8)$ & 0.5 \\
\hline O7 & $1.2327(3)$ & $-0.0046(3)$ & $0.9760(1)$ & $0.0311(6)$ & \\
\hline H7A & 1.2745 & -0.032 & 0.9413 & $0.047 *$ & \\
\hline H7B & 1.2277 & 0.0686 & 0.9823 & $0.047 *$ & \\
\hline S1 & $0.5257(1)$ & $0.85690(8)$ & $0.8389(4)$ & $0.0294(2)$ & \\
\hline S2 & $1.1467(2)$ & $-0.3568(1)$ & $0.5972(4)$ & $0.0442(3)$ & \\
\hline Mn1 & 1 & 0 & 1 & $0.0227(2)$ & \\
\hline 08 & $0.5535(3)$ & $1.1354(2)$ & $0.9399(1)$ & $0.0447(7)$ & \\
\hline H8A & 0.6146 & 1.1074 & 0.9659 & $0.067 *$ & \\
\hline H8B & 0.5616 & 1.0721 & 0.9191 & $0.067 *$ & \\
\hline O9 & $-0.4426(4)$ & $-0.3526(3)$ & $0.1307(2)$ & $0.074(1)$ & \\
\hline H9A & -0.3825 & -0.3419 & 0.1517 & $0.111^{*}$ & \\
\hline H9B & -0.4814 & -0.2857 & 0.1099 & $0.111^{*}$ & \\
\hline 010 & $0.5546(2)$ & $0.4314(2)$ & $0.76702(9)$ & $0.088(2)$ & \\
\hline H10A & 0.5831 & 0.4823 & 0.7476 & $0.132 *$ & \\
\hline
\end{tabular}




\begin{tabular}{l|l|l|l|l|l}
\hline H10B & 0.5819 & 0.3633 & 0.7461 & $0.132^{*}$ & \\
\hline O11 & $0.2477(3)$ & $1.2320(2)$ & $0.9845(1)$ & $0.159(4)$ & \\
\hline H11A & 0.3407 & 1.1949 & 0.9861 & $0.239^{*}$ & \\
\hline H11B & 0.2276 & 1.2548 & 0.9493 & $0.239^{*}$ & \\
\hline O12 & $0.6479(2)$ & $0.6008(2)$ & $0.70792(9)$ & $0.130(3)$ & \\
\hline H12B & 0.6624 & 0.6257 & 0.6732 & $0.195^{*}$ & \\
\hline H12A & 0.6128 & 0.6637 & 0.7324 & $0.195^{*}$ & \\
\hline O13 & $0.6131(4)$ & $-0.3953(2)$ & $0.5942(2)$ & $0.107(3)^{*}$ & 0.5 \\
\hline H13B & 0.6391 & -0.3935 & 0.5573 & $0.16^{*}$ & 0.5 \\
\hline H13A & 0.656 & -0.363 & 0.6145 & $0.16^{*}$ & 0.5 \\
\hline O15 & $0.8695(3)$ & $-0.4201(2)$ & $0.5154(2)$ & $0.077(2)^{*}$ & 0.5 \\
\hline H15A & 0.7977 & -0.4326 & 0.5049 & $0.115^{*}$ & 0.5 \\
\hline H15B & 0.9425 & -0.4495 & 0.4884 & $0.115^{*}$ & 0.5 \\
\hline O14 & $0.7365(4)$ & $-0.3399(2)$ & $0.5915(2)$ & $0.070(2)^{*}$ & 0.5 \\
\hline H14A & 0.7218 & -0.3823 & 0.5655 & $0.106^{*}$ & 0.5 \\
\hline H14B & 0.8103 & -0.3234 & 0.5808 & $0.106^{*}$ & 0.5 \\
\hline O16 & $0.5871(4)$ & $-0.4566(2)$ & $0.5298(2)$ & $0.092(3)^{*}$ & 0.5 \\
\hline H16A & 0.5231 & -0.4781 & 0.5171 & $0.137^{*}$ & 0.5 \\
\hline H16B & 0.6257 & -0.5054 & 0.5581 & $0.137^{*}$ & 0.5 \\
\hline H6 & $0.985(1)$ & $-0.412(7)$ & $0.589(2)$ & $0.137^{*}$ & 0.5 \\
\hline H6B & $0.958(9)$ & $-0.373(5)$ & $0.590(2)$ & $0.137^{*}$ & 0.5 \\
\hline H1N & $0.193(1)$ & $-0.107(9)$ & $0.461(4)$ & $0.137^{*}$ & \\
\hline & & & & & \\
\hline
\end{tabular}

$U_{e q}=\frac{1}{3}\left[U_{11}\left(a a^{*}\right)^{2}+U_{22}\left(b b^{*}\right)^{2}+U_{33}\left(c c^{*}\right)^{2}+2 U_{12} a b a^{*} b^{*} \cos \gamma+2 U_{13} a c a^{*} c^{*} \cos \beta\right.$

$$
+2 U_{23} b c b^{*} c^{*} \cos \alpha
$$

$U_{\text {iso }}=\exp \left[-8 \pi^{2} U\left(\sin ^{\theta} / \lambda\right)^{2}\right]$

Table S3 Anisotropic displacement parameters $\left(\mathrm{A}^{2}\right)$ for compound 1.

\begin{tabular}{l|l|l|l|l|l|l} 
Atoms & \multicolumn{1}{|c|}{$\boldsymbol{U}^{11}$} & \multicolumn{1}{|c|}{$\boldsymbol{U}^{22}$} & \multicolumn{1}{|c}{$\boldsymbol{U}^{33}$} & \multicolumn{1}{|c}{$\boldsymbol{U}^{12}$} & \multicolumn{1}{|c}{$\boldsymbol{U}^{13}$} & $\boldsymbol{U}^{23}$ \\
\hline C1 & $0.0238(18)$ & $0.0307(18)$ & $0.0208(16)$ & $-0.0060(15)$ & $-0.0018(13)$ & $0.0080(14)$ \\
\hline C2 & $0.031(2)$ & $0.0320(19)$ & $0.0196(16)$ & $-0.0026(16)$ & $-0.0024(14)$ & $0.0079(14)$ \\
\hline C3 & $0.032(2)$ & $0.0309(19)$ & $0.0230(17)$ & $-0.0035(16)$ & $-0.0028(14)$ & $0.0103(15)$ \\
\hline C4 & $0.0257(18)$ & $0.0284(18)$ & $0.0206(17)$ & $-0.0061(15)$ & $0.0006(13)$ & $0.0098(14)$ \\
\hline C5 & $0.0246(18)$ & $0.0254(17)$ & $0.0245(17)$ & $-0.0047(14)$ & $0.0009(13)$ & $0.0100(14)$ \\
\hline C6 & $0.0215(17)$ & $0.0279(18)$ & $0.0275(18)$ & $-0.0071(14)$ & $0.0021(13)$ & $0.0061(14)$ \\
\hline C7 & $0.031(2)$ & $0.0233(17)$ & $0.0320(19)$ & $-0.0068(15)$ & $-0.0070(15)$ & $0.0102(14)$ \\
\hline C8 & $0.037(2)$ & $0.0283(18)$ & $0.0251(18)$ & $-0.0127(16)$ & $-0.0032(15)$ & $0.0026(14)$ \\
\hline C9 & $0.0228(17)$ & $0.0274(18)$ & $0.0233(17)$ & $-0.0086(14)$ & $0.0008(13)$ & $0.0051(14)$ \\
\hline
\end{tabular}




\begin{tabular}{|c|c|c|c|c|c|c|}
\hline $\mathrm{C10}$ & $0.0254(18)$ & $0.0298(18)$ & $0.0210(16)$ & $-0.0073(15)$ & $-0.0012(13)$ & $0.0056(14)$ \\
\hline C11 & $0.0286(19)$ & $0.0242(18)$ & $0.0244(17)$ & $-0.0051(15)$ & $-0.0012(14)$ & $0.0049(14)$ \\
\hline $\mathrm{C} 12$ & $0.025(2)$ & $0.061(3)$ & $0.138(6)$ & $-0.010(2)$ & $-0.010(3)$ & $0.066(4)$ \\
\hline C13 & $0.030(3)$ & $0.059(3)$ & $0.146(6)$ & $-0.014(2)$ & $0.001(3)$ & $0.065(4)$ \\
\hline C14 & $0.0280(18)$ & $0.0222(16)$ & $0.0220(16)$ & $-0.0046(14)$ & $-0.0034(13)$ & 0.0075 (13) \\
\hline $\mathrm{C} 15$ & $0.025(2)$ & $0.033(2)$ & $0.068(3)$ & $-0.0065(17)$ & $-0.0080(19)$ & $0.022(2)$ \\
\hline C16 & $0.027(2)$ & $0.035(2)$ & $0.069(3)$ & $-0.0133(18)$ & $-0.0087(19)$ & $0.025(2)$ \\
\hline C17 & $0.032(2)$ & $0.0245(17)$ & 0.0215 (17) & $-0.0069(15)$ & $-0.0039(14)$ & $0.0075(14)$ \\
\hline C18 & $0.036(2)$ & $0.034(2)$ & $0.0290(19)$ & $-0.0131(17)$ & $-0.0039(16)$ & 0.0085 (16) \\
\hline C19 & $0.056(3)$ & $0.035(2)$ & $0.032(2)$ & $-0.022(2)$ & $-0.0188(19)$ & $0.0110(17)$ \\
\hline $\mathrm{C20}$ & $0.058(3)$ & $0.0247(18)$ & $0.0203(17)$ & $-0.0132(18)$ & $-0.0058(17)$ & $0.0061(14)$ \\
\hline $\mathrm{C21}$ & $0.046(2)$ & $0.031(2)$ & $0.0271(19)$ & $-0.0097(18)$ & $-0.0003(17)$ & $0.0037(15)$ \\
\hline $\mathrm{C22}$ & $0.037(2)$ & $0.033(2)$ & 0.0235 (18) & $-0.0082(17)$ & $-0.0024(15)$ & $0.0005(15)$ \\
\hline $\mathrm{C} 23$ & $0.230(11)$ & $0.047(3)$ & $0.065(4)$ & $-0.054(5)$ & $-0.048(5)$ & $0.018(3)$ \\
\hline $\mathrm{C24}$ & $0.200(9)$ & $0.037(3)$ & $0.052(3)$ & $-0.040(4)$ & $-0.038(4)$ & $0.019(2)$ \\
\hline $\mathrm{C25}$ & $0.048(3)$ & $0.032(2)$ & $0.046(2)$ & $-0.0062(19)$ & $0.019(2)$ & $0.0083(18)$ \\
\hline $\mathrm{C26}$ & $0.038(3)$ & $0.032(2)$ & $0.128(6)$ & $-0.008(2)$ & $-0.020(3)$ & $0.022(3)$ \\
\hline $\mathrm{C27}$ & $0.046(3)$ & $0.040(3)$ & $0.106(5)$ & $-0.015(2)$ & $-0.014(3)$ & $0.022(3)$ \\
\hline $\mathrm{C28}$ & $0.047(3)$ & $0.029(2)$ & $0.051(3)$ & $-0.0073(19)$ & $0.020(2)$ & 0.0051 (19) \\
\hline $\mathrm{C29}$ & $0.055(3)$ & $0.029(2)$ & $0.079(4)$ & $-0.008(2)$ & $0.006(3)$ & $-0.002(2)$ \\
\hline $\mathrm{C30}$ & $0.057(3)$ & $0.037(3)$ & $0.081(4)$ & $-0.004(2)$ & $-0.001(3)$ & $-0.001(3)$ \\
\hline C31 & $0.079(4)$ & $0.030(2)$ & $0.039(2)$ & $-0.012(2)$ & $0.024(2)$ & $0.0041(19)$ \\
\hline $\mathrm{C32}$ & $0.084(4)$ & $0.034(2)$ & $0.045(3)$ & $-0.019(3)$ & $0.023(3)$ & $0.001(2)$ \\
\hline $\mathrm{C33}$ & $0.068(4)$ & $0.044(3)$ & $0.076(4)$ & $-0.003(3)$ & $-0.015(3)$ & $0.020(3)$ \\
\hline $\mathrm{C} 34$ & $0.072(4)$ & $0.047(3)$ & $0.069(4)$ & $-0.004(3)$ & $-0.024(3)$ & $0.023(3)$ \\
\hline C35 & $0.030(2)$ & $0.043(2)$ & $0.055(3)$ & 0.0002 (19) & $0.008(2)$ & $0.021(2)$ \\
\hline $\mathrm{C36}$ & $0.059(3)$ & $0.057(3)$ & $0.078(4)$ & $-0.022(3)$ & $-0.012(3)$ & $0.035(3)$ \\
\hline C37 & $0.064(4)$ & $0.070(4)$ & $0.076(4)$ & $-0.027(3)$ & $-0.020(3)$ & $0.040(3)$ \\
\hline C38 & $0.032(2)$ & $0.051(3)$ & $0.051(3)$ & $-0.002(2)$ & $0.012(2)$ & $0.015(2)$ \\
\hline C39 & $0.047(3)$ & $0.051(3)$ & $0.055(3)$ & $-0.011(2)$ & $0.003(2)$ & $0.016(2)$ \\
\hline $\mathrm{C40}$ & $0.055(3)$ & $0.062(3)$ & $0.051(3)$ & $-0.014(3)$ & $0.006(2)$ & $0.011(3)$ \\
\hline $\mathrm{C41}$ & $0.056(3)$ & $0.054(3)$ & $0.079(4)$ & $-0.006(3)$ & $0.003(3)$ & $0.001(3)$ \\
\hline $\mathrm{C} 42$ & $0.048(3)$ & $0.049(3)$ & $0.069(3)$ & $-0.004(2)$ & $-0.001(3)$ & $0.011(3)$ \\
\hline N1 & $0.0218(14)$ & $0.0258(15)$ & $0.0196(14)$ & $-0.0026(12)$ & $0.0008(11)$ & $0.0056(11)$ \\
\hline $\mathbf{N 2}$ & $0.0247(15)$ & $0.0226(14)$ & $0.0186(14)$ & $-0.0039(12)$ & $0.0003(11)$ & $0.0064(11)$ \\
\hline N3 & $0.079(3)$ & $0.048(2)$ & $0.064(3)$ & $-0.026(2)$ & $0.001(2)$ & $0.011(2)$ \\
\hline N4 & $0.058(3)$ & $0.041(2)$ & $0.056(2)$ & $-0.016(2)$ & $0.018(2)$ & $-0.0080(19)$ \\
\hline N5 & $0.048(2)$ & $0.060(3)$ & $0.056(3)$ & $-0.012(2)$ & $0.009(2)$ & $-0.002(2)$ \\
\hline N6 & $0.046(2)$ & $0.060(3)$ & $0.068(3)$ & $-0.012(2)$ & $-0.008(2)$ & $0.021(2)$ \\
\hline
\end{tabular}




\begin{tabular}{l|l|l|l|l|l|l}
\hline O1 & $0.0454(18)$ & $0.0292(15)$ & $0.065(2)$ & $-0.0161(13)$ & $0.0007(15)$ & $-0.0043(14)$ \\
\hline O2 & $0.0521(18)$ & $0.0367(15)$ & $0.0225(13)$ & $-0.0022(14)$ & $0.0023(12)$ & $0.0076(11)$ \\
\hline O3 & $0.0346(15)$ & $0.0275(13)$ & $0.0316(14)$ & $-0.0033(11)$ & $0.0011(11)$ & $0.0073(11)$ \\
\hline O4 & $0.062(14)$ & $0.038(6)$ & $0.026(11)$ & $-0.019(8)$ & $-0.006(9)$ & $0.001(6)$ \\
\hline O5 & $0.062(6)$ & $0.040(5)$ & $0.019(4)$ & $-0.007(5)$ & $0.008(4)$ & $-0.015(3)$ \\
\hline O6 & $0.132(11)$ & $0.024(3)$ & $0.030(5)$ & $-0.042(4)$ & $-0.027(6)$ & $0.002(3)$ \\
\hline O4B & $0.064(15)$ & $0.030(5)$ & $0.018(4)$ & $-0.010(8)$ & $0.005(8)$ & $0.008(3)$ \\
\hline O5B & $0.137(15)$ & $0.075(12)$ & $0.053(8)$ & $0.047(9)$ & $-0.051(8)$ & $-0.023(7)$ \\
\hline O6B & $0.157(16)$ & $0.19(2)$ & $0.055(10)$ & $-0.145(17)$ & $0.013(10)$ & $-0.012(12)$ \\
\hline O7 & $0.0302(14)$ & $0.0332(14)$ & $0.0233(12)$ & $-0.0092(12)$ & $0.0041(10)$ & $0.0014(10)$ \\
\hline S1 & $0.0335(5)$ & $0.0205(4)$ & $0.0247(4)$ & $-0.0043(4)$ & $0.0020(3)$ & $0.0046(3)$ \\
\hline S2 & $0.0720(8)$ & $0.0315(5)$ & $0.0242(5)$ & $-0.0167(5)$ & $-0.0102(5)$ & $0.0031(4)$ \\
\hline Mn1 & $0.0220(4)$ & $0.0212(4)$ & $0.0170(4)$ & $-0.0023(3)$ & $-0.0010(3)$ & $0.0069(3)$ \\
\hline O8 & $0.0351(16)$ & $0.0542(19)$ & $0.0417(16)$ & $-0.0150(14)$ & $-0.0092(12)$ & $-0.0032(14)$ \\
\hline O9 & $0.063(3)$ & $0.053(2)$ & $0.100(3)$ & $-0.019(2)$ & $-0.006(2)$ & $-0.005(2)$ \\
\hline O10 & $0.119(4)$ & $0.072(3)$ & $0.070(3)$ & $-0.031(3)$ & $-0.048(3)$ & $0.027(2)$ \\
\hline O11 & $0.064(3)$ & $0.123(5)$ & $0.296(9)$ & $-0.061(3)$ & $0.081(4)$ & $-0.128(6)$ \\
\hline O12 & $0.197(7)$ & $0.125(5)$ & $0.095(4)$ & $-0.107(5)$ & $0.061(4)$ & $-0.061(4)$ \\
\hline
\end{tabular}

$U_{\mathrm{ij}}=\exp \left(-2 \pi^{2}\left[\mathrm{~h}^{2}\left(\mathrm{a}^{*}\right)^{2} \mathrm{U}_{11}+\mathrm{k}^{2}\left(\mathrm{~b}^{*}\right)^{2} \mathrm{U}_{22}+\mathrm{l}^{2}\left(\mathrm{c}^{*}\right)^{2} \mathrm{U}_{33}+2 \mathrm{hka} \mathrm{b}^{*} \mathrm{U}_{12} 2 \mathrm{hla}^{*} \mathrm{c}^{*} \mathrm{U}_{13}+2 \mathrm{klb} \mathrm{c}^{*} \mathrm{U}_{23}\right]\right)$

Table S4 Hydrogen bond parameters for compound 1 (distances in $\AA$ and angles in ${ }^{\circ}$ ).

\begin{tabular}{llllll}
\hline \multicolumn{1}{c}{$\mathrm{O}-\mathrm{H}$} & \multicolumn{1}{c}{$\mathrm{A}(\mathrm{O})$} & $\mathrm{O}-\mathrm{H}(\AA)$ & $\mathrm{H} \cdots \mathrm{A}(\AA)$ & $\mathrm{O} \cdots \mathrm{A}(\AA)$ & $\mathrm{O}-\mathrm{H} \cdots \mathrm{A}\left({ }^{\circ}\right)$ \\
\hline $\mathrm{O}(7)-\mathrm{H}(7 \mathrm{~A})$ & $\mathrm{O}(3)(-1+x, 1+y, z)$ & 0.82 & 1.90 & $2.723(4)$ & 178 \\
$\mathrm{O}(7)-\mathrm{H}(7 \mathrm{~B})$ & $\mathrm{O}(11)(-1+x, y, z)$ & 0.82 & 1.94 & $2.740(4)$ & 167 \\
$\mathrm{O}(8)-\mathrm{H}(8 \mathrm{~A})$ & $\mathrm{O}(7)(-x, 2-y,-z)$ & 0.82 & 2.00 & $2.809(4)$ & 169 \\
$\mathrm{O}(11)-\mathrm{H}(11 \mathrm{~A})$ & $\mathrm{O}(8)$ & 0.82 & 2.03 & $2.759(4)$ & 147 \\
$\mathrm{O}(9)-\mathrm{H}(9 \mathrm{~A})$ & $\mathrm{N}(3)(1-x, 1-y, 1-z)$ & 0.83 & 1.83 & $2.657(7)$ & 176 \\
$\mathrm{O}(9)-\mathrm{H}(9 \mathrm{~B})$ & $\mathrm{O}(8)$ & 0.83 & 1.89 & $2.720(4)$ & 175 \\
$\mathrm{O}(10)-\mathrm{H}(10 \mathrm{~B})$ & $\mathrm{N}(6)(1-x, 1-y, 1-z)$ & 0.83 & 2.03 & $2.823(5)$ & 160 \\
$\mathrm{O}(10)-\mathrm{H}(10 \mathrm{~A})$ & $\mathrm{O}(12)$ & 0.83 & 1.87 & $2.692(3)$ & 177 \\
$\mathrm{O}(12)-\mathrm{H}(12 \mathrm{~A})$ & $\mathrm{O}(13)$ & 0.82 & 1.83 & $2.540(4)$ & 145 \\
$\mathrm{O}(12)-\mathrm{H}(12 \mathrm{~A})$ & $\mathrm{O}(14)$ & 0.82 & 1.97 & $2.766(4)$ & 163 \\
$\mathrm{O}(13)-\mathrm{H}(13 \mathrm{~A})$ & $\mathrm{O}(12)$ & 0.83 & 2.07 & $2.540(4)$ & 123 \\
$\mathrm{O}(13)-\mathrm{H}(13 \mathrm{~B})$ & $\mathrm{O}(15)$ & 0.82 & 2.22 & $2.787(5)$ & 126 \\
$\mathrm{O}(15)-\mathrm{H}(15 \mathrm{~A})$ & $\mathrm{O}(13)$ & 0.82 & 2.42 & $2.787(5)$ & 108 \\
$\mathrm{O}(15)-\mathrm{H}(15 \mathrm{~A})$ & $\mathrm{O}(16)$ & 0.82 & 2.19 & $2.936(5)$ & 150
\end{tabular}




$\begin{array}{llllll}\mathrm{O}(12)-\mathrm{H}(12 \mathrm{~B}) & \mathrm{O}(2) & 0.82 & 1.94 & 2.752(4) & 169 \\ \mathrm{O}(14)-\mathrm{H}(14 \mathrm{~B}) & \mathrm{O}(6)(x,-1+y, z) & 0.82 & 2.28 & 3.010(2) & 148 \\ \mathrm{O}(14)-\mathrm{H}(14 \mathrm{~B}) & \mathrm{O}(6 \mathrm{~B})(x,-1+y, z) & 0.82 & 2.01 & 2.770(3) & 153 \\ \mathrm{O}(16)-\mathrm{H}(16 \mathrm{~A}) & \mathrm{O}(5) & 0.83 & 2.26 & 2.667(2) & 111 \\ \mathrm{O}(16)-\mathrm{H}(16 \mathrm{~A}) & \mathrm{O}(5 \mathrm{~B})(1+x,-1+y, z) & 0.83 & 2.35 & 2.820(3) & 117 \\ \mathrm{O}(6)-\mathrm{H}(6) & \mathrm{O}(15)(x, 1+y, z) & 0.86 & 2.35 & 2.745(2) & 109 \\ \mathrm{O}(6)-\mathrm{H}(6 \mathrm{~B}) & \mathrm{O}(14)(x, 1+y, z) & 1.15 & 1.99 & 3.010(2) & 145 \\ \mathrm{O}(6)-\mathrm{H}(6 \mathrm{~B}) & \mathrm{O}(15)(x, 1+y, z) & 1.15 & 2.16 & 2.745(2) & 108 \\ \mathrm{~N}(5)-\mathrm{H}(1 \mathrm{~N}) & \mathrm{N}(4) & 1.28 & 1.46 & 2.741(7) & 171\end{array}$

Table S5 Substrate overview for all the catalytic reactions.

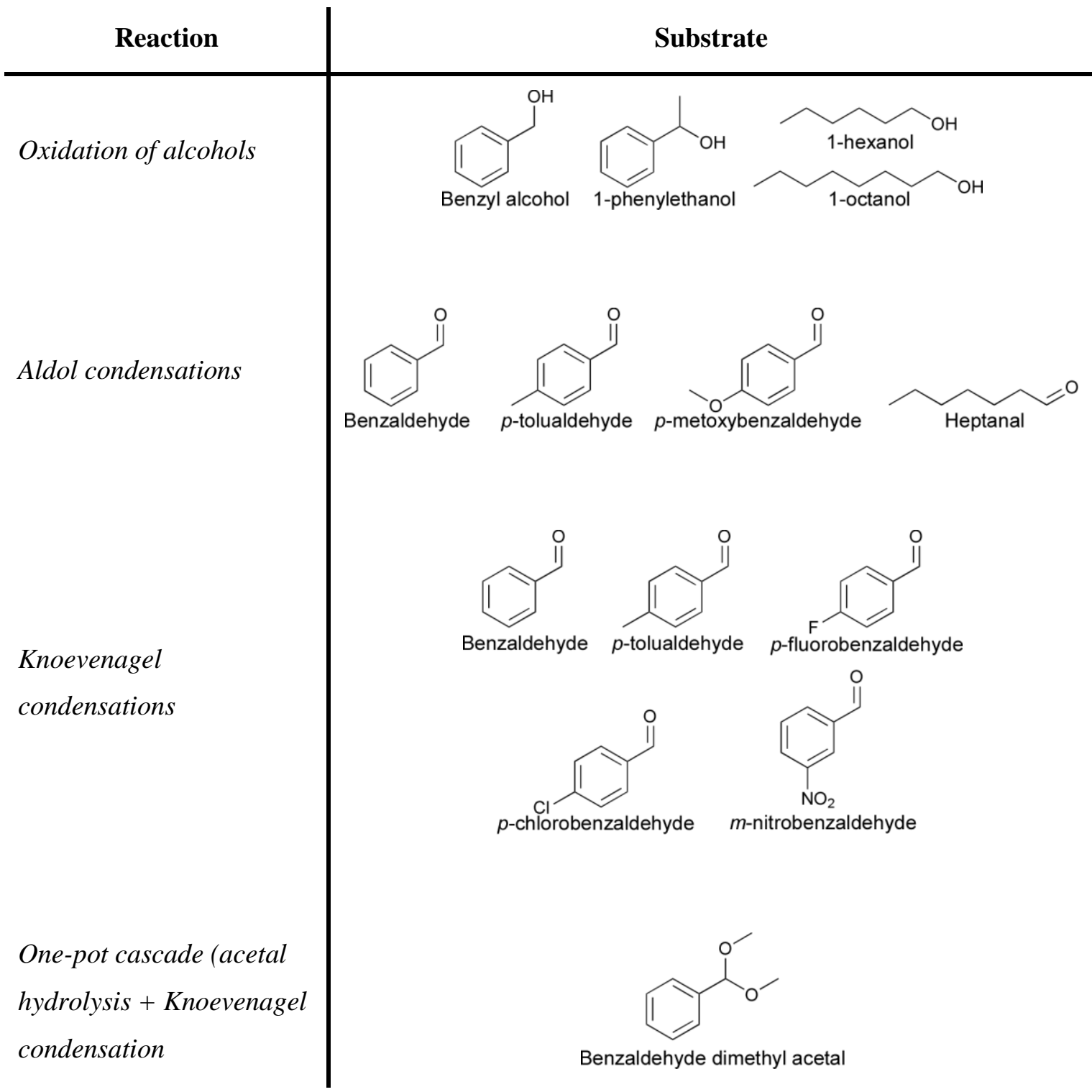


Table S6 Recyclability of the compound $\mathbf{1}$ and $\mathbf{3}$ for benzyl alcohol oxidation.

\begin{tabular}{cccc}
\multicolumn{2}{c}{ Compound 1 } & \multicolumn{2}{c}{ Compound 3 } \\
\hline Cycles & $\mathbf{C}_{\mathbf{T}}$ (4h) & Cycles & $\mathbf{C}_{\mathbf{T}} \mathbf{( 4 h )}$ \\
\hline 1 & $40 \%$ & 1 & $27.31 \%$ \\
2 & $31.3 \%$ & 2 & $31.42 \%$ \\
3 & $30.69 \%$ & 3 & $32 \%$ \\
4 & $26.8 \%$ & 4 & $26.8 \%$ \\
5 & $26.4 \%$ & 5 & $26 \%$ \\
& \multicolumn{2}{c}{$\mathrm{C}_{\mathrm{T}}=$ Total conversion }
\end{tabular}

Table S7 Recyclability of the compound $\mathbf{2}$ for benzaldehyde condensation.

Compound 2

\begin{tabular}{cc}
\hline Cycles & C $_{\mathbf{T}}$ (4h) \\
\hline 1 & $34.46 \%$ \\
2 & $33.07 \%$ \\
3 & $33.95 \%$ \\
4 & $25.60 \%$ \\
5 & $21.03 \%$
\end{tabular}

